\title{
UVUDF: UV Luminosity Functions at the Cosmic High Noon
}

\author{
Vihang Mehta ${ }^{1}$, Claudia Scarlata ${ }^{1}$, Marc Rafelski ${ }^{2,3,11}$, Timothy Gburek ${ }^{4}$, Harry I. Teplitz ${ }^{5}$, Anahita Alavi ${ }^{4}$, \\ Michael Boylan-Kolchin ${ }^{6}$, Steven Finkelstein ${ }^{6}$, Jonathan P. Gardner ${ }^{3}$, Norman Grogin ${ }^{2}$, Anton Koekemoer ${ }^{2}$, Peter Kurczynski ${ }^{7}$, \\ Brian Siana $^{4}$, Alex Codoreanu ${ }^{8}$, Duilia F. de Mello ${ }^{3,9}$, Kyoung-Soo Lee ${ }^{10}$, and Emmaris Soto ${ }^{9}$ \\ ${ }^{1}$ Minnesota Institute for Astrophysics, University of Minnesota, Minneapolis, MN 55455, USA \\ 2 Space Telescope Science Institute, Baltimore, MD 21218, USA \\ ${ }^{3}$ Goddard Space Flight Center, Code 665, Greenbelt, MD, 20771, USA \\ ${ }^{4}$ Department of Physics and Astronomy, University of California Riverside, Riverside, CA 92521, USA \\ ${ }^{5}$ Infrared Processing and Analysis Center, Caltech, Pasadena, CA 91125, USA \\ ${ }^{6}$ Department of Astronomy, The University of Texas at Austin, Austin, TX 78712, USA \\ ${ }^{7}$ Department of Physics and Astronomy, Rutgers, The State University of New Jersey, Piscataway, NJ 08854, USA \\ ${ }^{8}$ Centre for Astrophysics and Supercomputing, Swinburne University of Technology, Melbourne, Australia \\ ${ }^{9}$ The Catholic University of America, Physics Department, Washington DC, 20064, USA \\ ${ }^{10}$ Department of Physics, Purdue University, West Lafayette, IN 47906, USA \\ Received 2016 December 8; revised 2017 February 9; accepted 2017 February 20; published 2017 March 21
}

\begin{abstract}
We present the rest-1500 $\AA$ UV luminosity functions (LF) for star-forming galaxies during the cosmic high noonthe peak of cosmic star formation rate at $1.5<z<3$. We use deep NUV imaging data obtained as part of the Hubble Ultra-Violet Ultra Deep Field (UVUDF) program, along with existing deep optical and NIR coverage on the HUDF. We select F225W, F275W, and F336W dropout samples using the Lyman break technique, along with samples in the corresponding redshift ranges selected using photometric redshifts, and measure the rest-frame UV $\mathrm{LF}$ at $z \sim 1.7,2.2,3.0$, respectively, using the modified maximum likelihood estimator. We perform simulations to quantify the survey and sample incompleteness for the UVUDF samples to correct the effective volume calculations for the LF. We select galaxies down to $M_{\mathrm{UV}}=-15.9,-16.3,-16.8$ and fit a faint-end slope of $\alpha=-1.20_{-0.13}^{+0.10},-1.32_{-0.14}^{+0.10},-1.39_{-0.12}^{+0.08}$ at $1.4<z<1.9,1.8<z<2.6$, and $2.4<z<3.6$, respectively. We compare the star formation properties of $z \sim 2$ galaxies from these UV observations with results from $\mathrm{H} \alpha$ and UV + IR observations. We find a lack of high-SFR sources in the UV LF compared to the H $\alpha$ and UV+IR, likely due to dusty SFGs not being properly accounted for by the generic $I R X-\beta$ relation used to correct for dust. We compute a volume-averaged UV-to-H $\alpha$ ratio by abundance matching the rest-frame UV LF and $\mathrm{H} \alpha \mathrm{LF}$. We find an increasing UV-to-H $\alpha$ ratio toward low-mass galaxies $\left(M_{\star} \lesssim 5 \times 10^{9} M_{\odot}\right)$. We conclude that this could be due to a larger contribution from starbursting galaxies compared to the high-mass end.
\end{abstract}

Key words: galaxies: evolution - galaxies: high-redshift - galaxies: luminosity function, mass function galaxies: star formation

\section{Introduction}

The galaxy luminosity function (LF) is one of the key observables in astronomy, providing the number density of galaxies at a given luminosity and time. The LF is instrumental in establishing the connection between the observable light and the underlying distribution of dark-matter halos. The link between these two depends on the baryonic physics, which ultimately regulates the conversion of gas into stars and the luminosity output at any given wavelength.

In the rest-frame ultraviolet (UV), the galaxy continuum is dominated by light coming from young stars, and is therefore a direct tracer of recent star formation activity. Consequently, the UV LF can be used to describe the volume-averaged star formation rate in the universe and to study the in situ build up of stellar mass in galaxies. Moreover, unlike other star formation indicators, the rest-frame UV is continuously accessible to very high redshifts and hence is an invaluable diagnostic for mapping star formation out to very early times.

A large amount of effort has been devoted to obtaining accurate measurements of the rest-frame UV LF at all redshifts $z \lesssim 10$ (e.g., Arnouts et al. 2005; Sawicki \& Thompson 2006;

\footnotetext{
${ }^{11}$ NASA Postdoctoral Fellow.
}

Yoshida et al. 2006; Bouwens et al. 2007; Dahlen et al. 2007; Reddy \& Steidel 2009; Hathi et al. 2010; Oesch et al. 2010; van der Burg et al. 2010; Sawicki 2012; Alavi et al. 2014, 2016; Bouwens et al. 2014a, 2014b, 2015; Finkelstein et al. 2015; Bernard et al. 2016; Parsa et al. 2016). These observations show that the UV luminosity density increases steadily up to $z \sim 2-3$, followed by a slight decline out to the highest redshifts probed so far (e.g., see Alavi et al. 2016).

Recently, very faint galaxies have attracted significant attention for a variety of reasons. At $z \sim 6-10$, they are expected to be essential for reionization of the universe (Bouwens et al. 2012; Finkelstein et al. 2012; Jaacks et al. 2012; Robertson et al. 2013, 2015), and they include likely progenitors of $L^{\star}$ galaxies in the local universe (e.g., Boylan-Kolchin et al. 2015). At intermediate redshifts, faint galaxies provide excellent tests of feedback due to star formation and reionization (e.g., Benson et al. 2003; Lo Faro et al. 2009; Weinmann et al. 2012). In the nearby universe, these systems probe galaxy formation on the finest scales and may contain clues to the nature of dark matter (e.g., Menci et al. 2012, 2016; Nierenberg et al. 2013; Kennedy et al. 2014). The evolution of the faint-end slope $(\alpha)$ of the UV LF can therefore inform us on many crucial aspects of galaxy formation and evolution. 
It is not surprising then that the value of $\alpha$ and its time evolution have been the subject of much research, and it is highly debated in the current literature. From published results, $\alpha$ appears to evolve dramatically, going from $\alpha \sim-1.2$ at $z \sim 0$ to $\alpha \sim-2$ by $z \sim 8$, albeit with a rather large scatter. At $z \sim 2$, the faint-end slope estimates vary from considerably shallow values of $\alpha \sim-1.3$ (Hathi et al. 2010; Parsa et al. 2016) to very steep values of $\alpha=-1.72$ (Alavi et al. 2014, 2016). The survey limits are the main challenge in accessing the faint galaxies needed to significantly constrain the value of $\alpha$ (e.g., Oesch et al. 2010). Strong gravitational lensing enables one to circumvent this limitation, although it introduces additional systematics and complications, such as a non-trivial effective survey volume calculation (e.g., Alavi et al. 2014, 2016). Deep, direct imaging still provides the most robust estimate for $\alpha$.

Complementary to the $\mathrm{UV}$, the $\mathrm{H} \alpha$ recombination line is a gold-standard indicator for ongoing star formation. These two tracers, however, are sensitive to star formation occurring over different timescales ${ }^{12}$ (Kennicutt \& Evans 2012), and are affected differently by interstellar dust attenuation. In the local universe, the two indicators are found to agree with each other, under the assumption that the star formation has been constant over a long enough time to allow equilibrium ( $>100 \mathrm{Myr}$; e.g., Buat et al. 1987; Buat 1992; Bell \& Kennicutt 2001; IglesiasPáramo et al. 2004; Salim et al. 2007; Lee et al. 2009; Barnes et al. 2011; Hermanowicz et al. 2013). The effect of dust attenuation in the rest-frame UV is usually corrected using locally calibrated empirical relations between the slope of the UV continuum and the IR excess (IRX- $\beta$ relation; Meurer et al. 1999). The Meurer et al. (1999) relation was calibrated for central starbursts in the nearby universe. On the whole, starforming galaxies in the nearby universe lie below this relation, as found by many studies (e.g., Muñoz-Mateos et al. 2009; Boquien et al. 2012; Grasha et al. 2013; Ye et al. 2016).

At high redshifts, it has been suggested that star formation is dominated by more stochastic, intense bursts, which may also be more important in low(er)-mass galaxies (e.g., Shen et al. 2013; Hopkins et al. 2014; Domínguez et al. 2015). If this is true, the constant star formation history (SFH) assumption implicit in all luminosity-to-star formation rate conversions breaks down. There are also indications that the Meurer et al. (1999) correction for dust may not be adequate at $z \gtrsim 1$ (e.g., Buat et al. 2012; Dayal \& Ferrara 2012; Wilkins et al. 2012; Castellano et al. 2014; de Barros et al. 2014; Reddy et al. 2015; Smit et al. 2015; Talia et al. 2015; ÁlvarezMárquez et al. 2016). Until JWST comes online, the highest possible redshift where a direct comparison of the two SFR indicators can be performed is $z=2.5$.

In this paper, we use the Ultra-Violet Ultra Deep Field (UVUDF; Teplitz et al. 2013), which is among the deepest UV data ever obtained, to derive the rest-frame UV LF at $z \sim 1.7,2.2,3$, and constrain its faint-end slope. In addition, we use $\mathrm{H} \alpha$ LFs available from the literature to compare the volume-averaged SFR derived with the two SFR indicators at $z \sim 2$. This paper is organized as follows: Section 2 describes the UVUDF data used in this work as well as our sample selection, Section 3 describes the completeness simulations and

\footnotetext{
${ }^{12}$ The $\mathrm{H} \alpha$ emission traces star formation over short timescales $(\sim 10 \mathrm{~s}$ of $\mathrm{Myr}$, typical of the hot, O- and B-type stars that power $\mathrm{H}$ II regions). On the other hand, the contribution to the rest-frame UV continuum comes from the longerlived B-A stars $(\sim 100 \mathrm{Myr})$.
}

presents the selection functions, Section 4 outlines the LF fitting procedure as well as our results, Section 5 presents our results, Section 6 discusses our results in the context of recent literature and analyzes the implications, and Section 7 summarizes our conclusions. Throughout this paper, we assume cosmological parameters from Table 3 of Planck Collaboration et al. (2015): $\Omega_{m}=0.315, \Omega_{\lambda}=0.685$, and $H_{0}=67.31 \mathrm{~km} \mathrm{~s}^{-1} \mathrm{Mpc}^{-1}$, and all magnitudes used are $\mathrm{AB}$ magnitudes (Oke \& Gunn 1983).

\section{Data and Sample Selection}

\subsection{UVUDF Data}

The full UVUDF data set is comprised of 11 photometric broadband filters covering the Hubble UDF $(\alpha(J 2000)=$ $0.3^{\mathrm{h}} 32^{\mathrm{m}} 39^{\mathrm{s}}, \delta(J 2000)=-27^{\circ} 47^{\prime} 39$ !! 1$) \quad$ spanning wavelengths from the NUV to NIR. The NUV coverage of the HUDF provided by the UVUDF observations includes three WFC3-UVIS filters: F225W, F275W, and F336W (Teplitz et al. 2013). The optical wavelengths are covered by four ACS filters: F435W, F606W, F775W, and F850LP (Beckwith et al. 2006). The NIR is covered by four WFC3-IR filters: F105W, F125W, F140W, and F160W, obtained as part of the UDF09 and UDF12 programs (Oesch et al. 2010; Bouwens et al. 2011; Ellis et al. 2013; Koekemoer et al. 2013). Moreover, the entire field is also covered in F105W, F125W, and F160W as part of the CANDELS GOODS-S observations (Grogin et al. 2011; Koekemoer et al. 2011). The UVUDF field with coverage in all 11 filters covers an area of $7.3 \mathrm{arcmin}^{2}$. The data reduction, photometry, and source catalog generation for the UVUDF is fully developed and described in Rafelski et al. (2015). We use the final catalog provided by Rafelski et al. (2015) for this work.

Before applying the sample selection cuts, we remove all sources that are flagged as stars in the UVUDF catalog. Furthermore, we flag bright, compact sources $\left(z_{850}<25.5\right.$ and half-light radii, $r_{1 / 2}<1^{\prime \prime}$ ) with a SExtractor stellarity parameter $>0.8$ as stars. This criterion is only reliable for bright sources and hence, we instead use a color-color cut based on the Pickles (1998) stellar library at fainter magnitudes to flag stars. At $z_{850}>25.5$, we flag compact sources $\left(r_{1 / 2}<1^{\prime \prime}\right)$ that have $V-i$ versus $i-z$ colors consistent with the Pickles (1998) stellar sequence to within 0.15 mag as stars. Lastly, we confirm that no stars are left in the final samples by visual inspection.

\subsection{Dropouts Sample Selection}

The Lyman break feature in galaxy SEDs has been proven to be very efficient at selecting high-redshift galaxies (e.g., Steidel et al. 1996, 1999, 2003; Adelberger et al. 2004; Bouwens et al. 2004, 2006, 2010, 2011; Bunker et al. 2004, 2010; Rafelski et al. 2009; Reddy \& Steidel 2009; Oesch et al. 2010; Hathi et al. 2012; Reddy et al. 2012). Here, we use the NUV filters available in the UVUDF to identify the Lyman break galaxies (LBGs) in the redshift range of $z \sim 1.5-3.5$.

The LBG dropout selection criteria we use are based on standard color-color and $\mathrm{S} / \mathrm{N}$ cuts, similar to Hathi et al. (2010), Oesch et al. (2010), and Teplitz et al. (2013). However, we further optimize the $\mathrm{S} / \mathrm{N}$ cuts using the mock galaxy sample generated for our completeness simulations (see Section 3). The color-color selection criteria are shown in Figure 1. Specifically, we select galaxies between $z \sim 1.4-1.9$ 

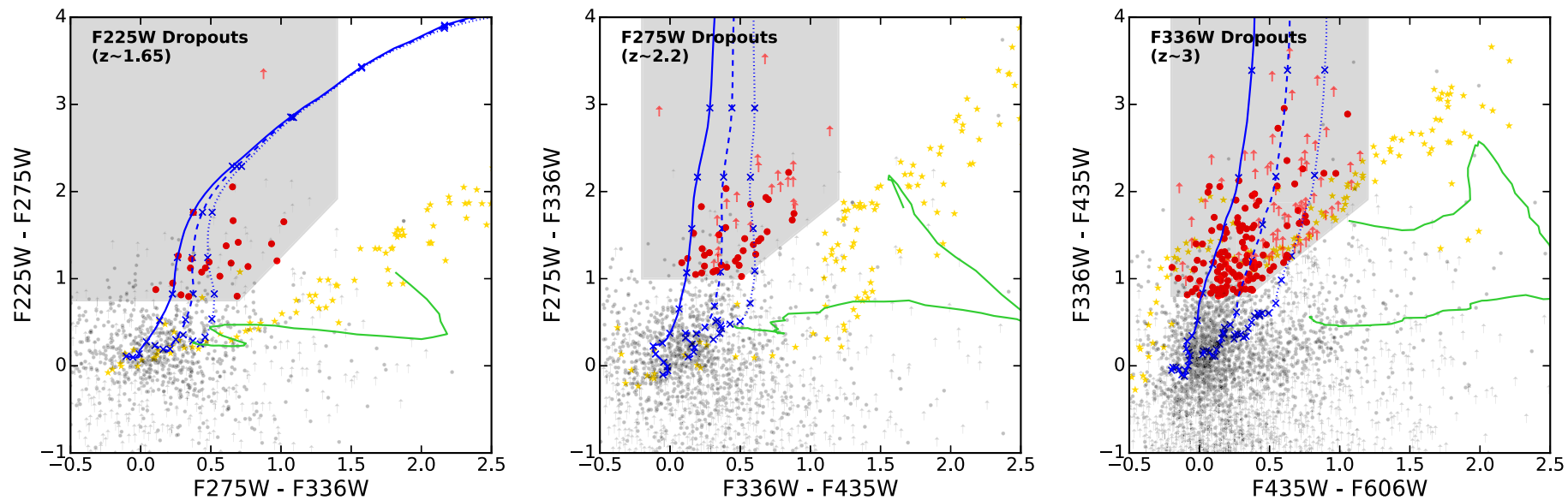

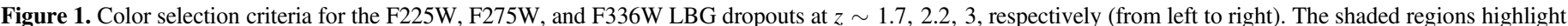

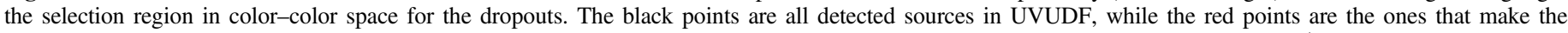

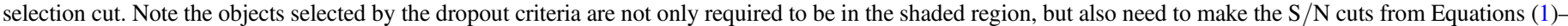

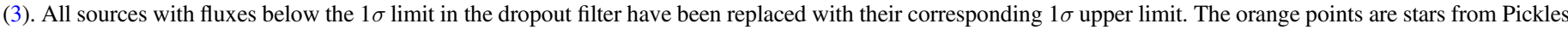

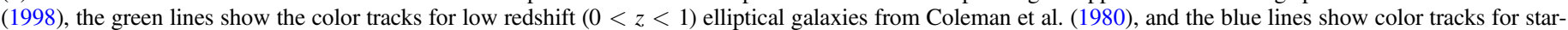

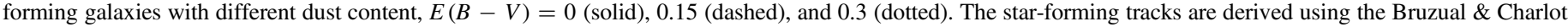
(2003) template for constant star formation rate, solar metallicity, age of $100 \mathrm{Myr}$, and dust extinction defined by the Calzetti et al. (2000) law.

as follows:

$$
\left\{\begin{array}{l}
\text { F225W }-\mathrm{F} 275 \mathrm{~W}>0.75 \\
\text { F275W }-\mathrm{F} 336 \mathrm{~W}>-0.5 \\
\text { F275W }-\mathrm{F} 336 \mathrm{~W}<1.4 \\
\mathrm{~F} 225 \mathrm{~W}-\mathrm{F} 275 \mathrm{~W}>[1.67 \times(\mathrm{F} 275 \mathrm{~W}-\mathrm{F} 336 \mathrm{~W})]-0.42 \\
\mathrm{~F} 336 \mathrm{~W}-\mathrm{F} 435 \mathrm{~W}>-0.5 \\
\mathrm{~S} / \mathrm{N}(\mathrm{F} 275 \mathrm{~W})>5
\end{array}\right.
$$

This results in a sample of 25 galaxies from the UVUDF catalog. Similarly, galaxies between $z \sim 1.8-2.6$ are selected using the following criteria:

$$
\left\{\begin{array}{l}
\text { F275W }- \text { F336W }>1.0 \\
\text { F336W }- \text { F435W }>-0.2 \\
\text { F336W }- \text { F435W }<1.2 \\
\text { F275W }- \text { F336W }>[1.3 \times(F 336 W-F 435 W)]+0.35 \\
S / N(F 336 W)>5 \\
S / N(F 225 W)<1
\end{array}\right.
$$

providing a sample of 60 galaxies. The galaxies between $z \sim 2.4-3.6$ are selected as

$$
\left\{\begin{array}{l}
\text { F336W }- \text { F435W }>0.8 \\
\text { F435W }- \text { F606W }>-0.2 \\
\text { F435W }- \text { F606W }<1.2 \\
\text { F336W }- \text { F435W }>[1.3 \times(F 435 W-F 606 W)]+0.35 \\
\mathrm{~S} / \mathrm{N}(\mathrm{F} 435 \mathrm{~W})>5 \\
\mathrm{~S} / \mathrm{N}(\mathrm{F} 275 \mathrm{~W})<1
\end{array}\right.
$$

which returns 228 galaxies. When applying these color selection criteria, all sources with magnitudes below the $1 \sigma$ limit in the dropout filter are replaced with their corresponding $1 \sigma$ upper limits, as determined from our completeness simulations (see Section 3).

\subsection{Photometric Redshift Sample Selection}

The inclusion of NUV data (in addition to the optical and NIR) enhances the photometric redshift accuracy, particularly at $z<0.5$ and $2<z<4$ (Rafelski et al. 2015). The UVUDF catalog includes photometric redshifts calculated using photometry from the 11 broadband filters via the Bayesian Photometric Redshift (BPZ) algorithm (Benéz 2000; Benéz et al. 2004; Coe et al. 2006). The SED templates used for BPZ are based on those from PEGASE (Fioc \& Rocca-Volmerange 1997) recalibrated using redshift information from FIREWORKS (Wuyts et al. 2008). The quality of the photometric redshift is reported by two quantities, ODDS (measuring the spread in the probability distribution function, $P(z)$ ) and modified reduced $\chi^{2}$ (measuring the goodness of fit). ${ }^{13}$ We require the photometric redshift sample to have ODDS $>0.9$ and modified reduced $\chi^{2}<1$ to ensure only sources with reliable photometric redshifts are selected. Applying these cuts gives a sample of 234, 258, and 440 galaxies in the redshift ranges $1.4<z<1.9,1.8<z<2.6$, and $2.4<z<$ 3.6 , respectively.

Using the photometric redshifts enables sample selection down to fainter magnitudes than the corresponding dropout criteria. The sample of dropout-selected galaxies requires a signal-to-noise of $>5 \sigma$ in the detection band to confirm the strength of the break. On the other hand, photometric redshiftselected samples only require a $5 \sigma$ detection in the rest- $1500 \AA$ filter. At these redshifts, the dropout detection band (F275W for F225W dropouts, F336W for F275W dropouts, F435W for F336W dropouts) is not the same as the rest- $1500 \AA$ filter (F435W for $z<2.2$; F606W for $z>2.2$ ). This is because the dropout detection band looks for flux immediately redward of the Ly $\alpha(1216 \AA)$, whereas the rest-frame UV flux is still redward at $1500 \AA$. Since the optical data available are deeper than NUV, the photometric redshift samples select galaxies down to fainter rest-1500 ̊ magnitudes.

\footnotetext{
${ }^{13}$ The modified reduced $\chi^{2}$ reported by BPZ is similar to a normal reduced $\chi^{2}$, except it includes an additional uncertainty for the SED templates in addition to the uncertainty in the galaxy photometry (Coe et al. 2006). The resultant $\chi^{2}$ is a more realistic measure of the quality of the fit (for a detailed discussion see Rafelski et al. 2009).
} 
We fit the LFs for both LBG samples as well as the photometric redshift-selected galaxy samples in the same redshift ranges as the dropout criteria to validate the fit robustness. Moreover, the depth of the photometric redshift sample allows for better constraints on the faint-end slope.

\section{Completeness}

Survey incompleteness and sample selection effects greatly impact the effective surveyed volume of a sample, a quantity critical to computing LFs. We need a precise estimate of the completeness for the UVUDF samples in order to properly and accurately correct the volume density. A common approach for completeness estimation in field galaxy studies (e.g., Oesch et al. 2010; Finkelstein et al. 2015) is to insert mock galaxies into real data, apply identical data reduction and sample selection, and analyze the fraction of recovered artificial galaxies as a function of galaxy properties such as magnitude, redshift, and galaxy size. We perform an extensive set of completeness simulations following a similar procedure in order to quantify the completeness for the UVUDF.

\subsection{Completeness Simulations}

We start by generating a set of mock galaxies with properties representative of the observed sample. These mock galaxies are then planted directly into the real science images, thus preserving the noise properties. Only 150 mock galaxies are inserted at a time to also preserve the crowding properties of the data. These images with artificial galaxies are then put through the same data reduction, analysis for source detection, photometry, photometric redshift, and sample selection as performed for the real data. By keeping track of the fraction of recovered and selected mock sources compared to the total number of input sources, we can quantify the completeness. Our full set of simulations consists of repeating this process for a total of 45,000 mock galaxies over 300 separate iterations.

To ensure that the mock galaxies used for our completeness simulations are consistent with the observed sample, we assign the absolute magnitudes for our mock galaxies according to existing prescriptions of the UV LFs from the literature. In particular, we use the rest-frame UV LFs from Oesch et al. (2010) to randomly generate a set of rest-1500 $\AA$ absolute magnitudes for our mock sample. The initial redshift distribution for our simulated sources is taken to be flat, i.e., $d N / d z$ is constant.

The colors for our mock sample are assigned using spectral templates from the Bruzual \& Charlot (2003, BC2003) models. Each mock galaxy is given a set of model parameters: metallicity, age, exponential SFR $\tau$, and dust extinction. The metallicity is chosen to be random from $Z / Z_{\odot}=0.0001$, 0.0004, 0.004, 0.008, and 0.02 (preset BC2003 models). We use the distributions of age, exponential SFR $\tau$, and dust extinction $(E(B-V))$ from observed galaxies in 3D-HST (Skelton et al. 2014) to randomly generate these parameters for our mock galaxies. We also include the contribution from nebular emission lines using line ratios from Anders \& Fritze-v. Alvensleben (2003). We apply a Calzetti et al. (2000) dust extinction law as well as the Inoue et al. (2014) IGM attenuation model to the SEDs to simulate the dust extinction and IGM absorption, respectively. After translating the SEDs to the appropriate redshift, the magnitudes for the rest of the filters are then obtained by computing the contribution of the SED in the particular filter according to its response curve.

We generate the mock galaxies for our completeness simulations using the IRAF task, mkobjects. The sizes for the mock galaxies are defined by assigning a half-light radius for each source. We use the observed distribution of $\mathrm{B}_{435}$-band sizes for all sources (no cuts applied) in the UVUDF to randomize the half-light radii for our mock galaxies. The distribution of simulated half-light radii is roughly representative of a log-normal with a peak at 2.7 pixels, with a tail toward larger radii, giving an interquantile range of 2.5-4.9 pixels corresponding to a physical size of $\sim 0.6-1.3 \mathrm{kpc}$ at $z \sim 2$. The observed UVUDF catalog shows no significant size bias (in pixels) as a function of redshift for $z<4$ and hence, we choose the size distribution of our mock galaxies to be uniform at all redshifts, in order to fully explore the parameter space. We choose the $\mathrm{B}_{435}$ band since it is the closest filter in wavelength and has a similar resolution to the rest-frame UV, in addition to the deep coverage available as well as the relatively narrow point-spread function (PSF).

To fully generate an artificial galaxy, mkobjects also requires a Sérsic index $(n)$, axial ratio, image position, and position angle. The mock galaxies are assigned a Sérsic profile that either represents exponential disks ( $n=1$; good description of spiral galaxies) or a de Vaucoleuers profile ( $n=4$; good description of elliptical galaxies). These represent the two extremes for light profiles of observed galaxies. We fix the probability for a mock galaxy to have $n=1$ or $n=4$ to be equal (50\% each). Although observed galaxies do not exhibit this distribution, completeness as a function of Sérsic index is expected to be well-behaved between the two extremes. Our choice is motivated by wanting to properly sample the two extremes. Furthermore, we verify the simulation output and confirm that the choice of this Sérsic index distribution does not bias the completeness in any statistically significant manner.

The ellipticities (axial ratios) for our mock sample are randomized using the distribution of the observed $\mathrm{B}_{435}$-band axial ratios in the UVUDF, with a peak at 0.7 and long tail toward lower axial ratios. The position of the simulated sources is randomized within a $4000 \times 4400$ pixel $\left(2^{\prime} \times 2\right.$ '.5) region that ensures UV coverage of the UDF. We further limit the positions of mock galaxies to avoid chip edges as well as the WFC3/UVIS chip gap, as is done for the real sample. By allowing for random positions, we can encapsulate any variations in the depth or noise properties across the imaging data. Lastly, the position angles are randomized between $0^{\circ}$ and $360^{\circ}$.

We determine the optimal number of galaxies to insert in one iteration in order to avoid crowding issues by planting a varying number of mock galaxies into the images. From this, we find that the scatter in the recovered photometry compared to the input does not increase significantly between 100 and 200 sources inserted per iteration. Thus, planting $\lesssim 200$ sources per iteration does not change the crowding properties of our field. Being conservative, we choose to insert 150 sources per iteration. This is similar to the treatment for completeness simulations done for the CANDELS/GOODS fields by Finkelstein et al. (2015).

We split the simulation into 100 separate iterations and for each iteration, we insert 150 mock galaxies into the original images. The mock galaxies are simulated and inserted into the 
original image for each of the 11 filters using mkobjects. The newly generated images with simulated sources are then run through identical data reduction, photometry, and the catalog creation process as for the real data.

Briefly, the process involves using Colorpro (Coe et al. 2006), which runs Source Extractor (Bertin \& Arnouts 1996) for all filters in dual-image mode, to measure photometry in the images. ColorPro also applies aperture corrections for the different aperture sizes as well as PSF corrections to account for variations in the PSF across filters. The detection image used is created from the four optical and four NIR filters to maximize the depth and robustness of the aperture sizes. In order to properly recover both bright and faint sources in a crowded field, photometry is measured using two different detection thresholds and two different deblending thresholds, which are then merged into a single photometric catalog. For the smaller apertures needed for the NUV filters, F435W is used as the detection image instead. The full methodology is described in detail in Rafelski et al. (2015).

The UVUDF catalog includes aperture-matched PSF-corrected photometry for a robust measurement of flux across images with varying PSFs. This is done by measuring photometry on highresolution data and applying a PSF correction for the NIR filters, which have larger PSFs. The PSF correction is determined by degrading the $\mathrm{I}_{775}$ image (reddest high-resolution image with a well-behaved PSF available) to each of the NIR filters using the IRAF task psfmatch. Instead of convolving the entire image after mock galaxies are inserted (which is computationally expensive), we instead create $500 \times 500$ px $\left(15^{\prime \prime} \times 15^{\prime \prime}\right)$ stamps for the simulated sources using mkobjects and convolve them individually before adding them to the PSF-matched image. This saves considerable amount of computation time per iteration.

Lastly, we generate a catalog using the images with mock galaxies following the same pipeline as used for the real UVUDF catalog (Rafelski et al. 2015) and compare it to the input catalog to determine the fraction of recovered objects. In order to be recovered, an object is required to be positionally matched within $3 \mathrm{px}\left(0{ }^{\prime \prime} 09\right)$.

Typically, incompleteness is computed as a function of magnitude and redshift; however, we also consider another key factor: galaxy size. Even at a constant magnitude and redshift, an extended galaxy may not be recovered due to the low surface brightness compared to a compact one. We correct the effective volumes for our sample according to the magnitude, redshift, as well as the galaxy size using selection functions as described in Section 3.2. We also compute the completeness as a function of apparent magnitude and galaxy size, which is used to define the survey magnitude limits (see Appendix A).

\subsection{Selection Functions}

The mock galaxy sample can be used to determine the probability of a galaxy to satisfy the LBG selection criteria as long as there are no biases in the recovered magnitudes and colors of the mock galaxies. Before proceeding, we verify that our completeness simulations do not introduce any offsets or biases in the magnitudes and colors of recovered mock galaxies. We apply the LBG selection criteria to the input mock sample to quantify the relative efficiency of the criteria to select a galaxy with a given redshift, rest-1500 $\AA$ absolute magnitude, and half-light radius. The top row in Figure 2 shows the selection function for the three dropout criteria from Section 2.2, marginalized over all galaxy sizes.
Similarly, the photometric redshift selection criteria are also applied to the mock catalog and the corresponding selection functions are derived for the photometric redshift samples. These are plotted in the bottom row of Figure 2, again marginalized over all galaxy sizes. These selection functions are used to compute the effective volumes when fitting the UV LF in Section 4.

The selection functions plotted in Figure 2 have been marginalized over all galaxy sizes. However, the information in the size dimension is preserved and the effective volume for each source is computed according to its size. The overall effect of galaxy sizes on the completeness can be visualized in Figure 9 in Appendix A, which shows the survey incompleteness as a function of observed magnitude and galaxy size.

\subsection{Redshift Distribution of the Dropout Sample}

The selection functions computed here, when marginalized over the magnitude and size dimensions, describe the redshift distribution of galaxies in the selected sample. As a validation check, we compare the selection functions from our simulations with redshifts measured from observations. Spectroscopic redshifts are available in the UVUDF catalog (Rafelski et al. 2015) for a small fraction of the sources in our sample, whereas photometric redshifts are available for all the sources in the UVUDF catalog. Figure 3 shows the distribution of spectroscopic (where available) as well as photometric (for all sources) redshifts. The selection functions derived from our completeness simulations are overplotted for comparison. As seen in the figure, the redshift distributions (shown as histograms) are in overall agreement with the selection functions (shown as curves). Note that the photometric redshifts and simulations are not expected to agree one-to-one by construction, because the galaxy templates used to estimate the photometric redshifts (Section 2.3) are not the same as those used to generate the mock catalog for our simulations (Section 3).

\section{Deriving the LF Parameters}

The rest-frame $1500 \AA$ UV LF is one of the key diagnostics for establishing the link between galaxy luminosities, galaxy masses, and the cosmic star formation rate. Considerable effort has been put into characterizing the shape and evolution of the UV LF near the peak of cosmic star formation $(z \sim 1-3)$ (e.g., Reddy \& Steidel 2009; Hathi et al. 2010; Oesch et al. 2010; Sawicki 2012; Alavi et al. 2014, 2016; Parsa et al. 2016). Here, we fit UV LFs using the dropout as well as photometric redshift-selected samples described in Section 2 corresponding to the three redshift ranges: $z \sim 1.7,2.2,3.0$.

Using the Schechter function (Schechter 1976) as the parametric shape for the UV LF is well motivated, as it matches the observed universe well:

$$
\phi(M)=0.4 \ln (10) \phi^{\star} 10^{-0.4\left(M-M^{\star}\right)(1+\alpha)} e^{-10^{-0.4\left(M-M^{\star}\right)}} .
$$

We perform a maximum likelihood analysis to fit the UV LFs. Specifically, we use the modified maximum likelihood estimator (MLE) developed and presented in Mehta et al. (2015), which accounts for the measurement errors in the galaxies' observed magnitude, allowing for a more robust fitting procedure. Following the procedure from Mehta et al. (2015), we define the probability for detecting a galaxy in the 

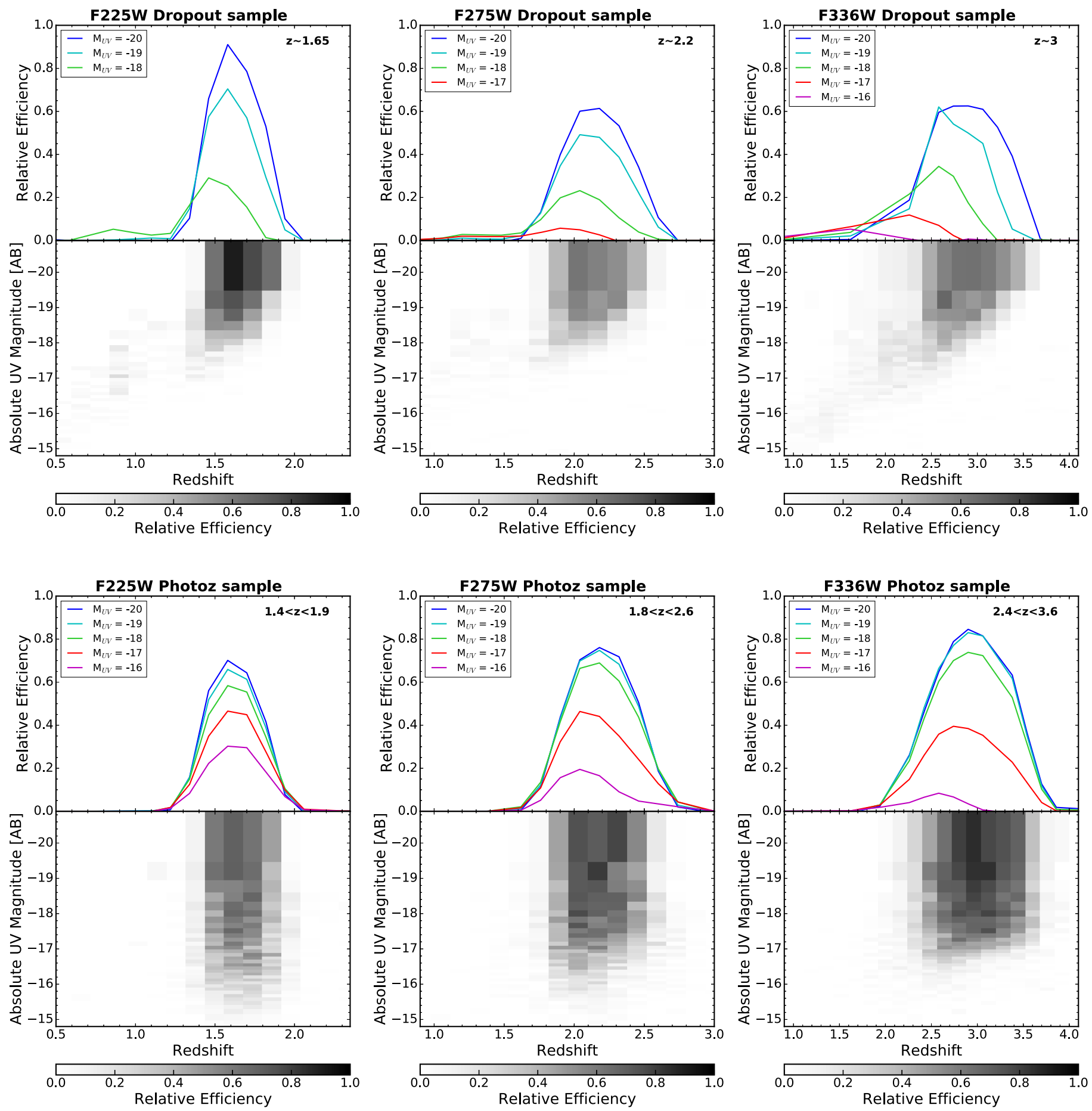

Figure 2. (Top row) Selection functions for the F225W, F275W, and F336W dropout criteria shown as a function of redshift (top panel) and as a function of both redshift and absolute rest-frame UV magnitude (bottom panel). This shows the redshift and absolute rest-frame UV magnitude distributions of galaxies selected by the dropout criteria. The relative efficiency is the fraction of sources that are selected from the full input sample after applying the selection cuts. (Bottom row) Selection functions for the photometric redshift samples corresponding to the redshift selected with the dropout criteria. Requiring a $5 \sigma$ detection in the observed rest-frame UV magnitude and applying the cuts to ensure good quality photometric redshifts (ODDS $>0.9$ and modified reduced $\left.\chi^{2}<1.0\right)$ are the primary factors affecting the relative efficiency of these selection functions.

sample as

$$
P\left(M_{i}\right)=\frac{\iint_{-\infty}^{M_{\text {lim }}(z)} \phi(M) \cdot v_{\mathrm{eff}}(M, z) .}{N\left(M \mid\left\{M_{i}, \sigma_{i}\right\}\right) d M d z} .
$$

Here, $M_{i}$ is the absolute rest- $1500 \AA$ (UV) magnitude of the galaxy, $\phi(M)$ is the LF from Equation (4), $M_{\lim }(z)$ represents the survey's detection limit, $v_{\text {eff }}(M, z)$ is the effective differential comoving volume, and $N\left(M \mid\left\{M_{i}, \sigma_{i}\right\}\right)$ is the term that marginalizes over the measurement error in the galaxy's magnitude. The effective differential comoving volume is 

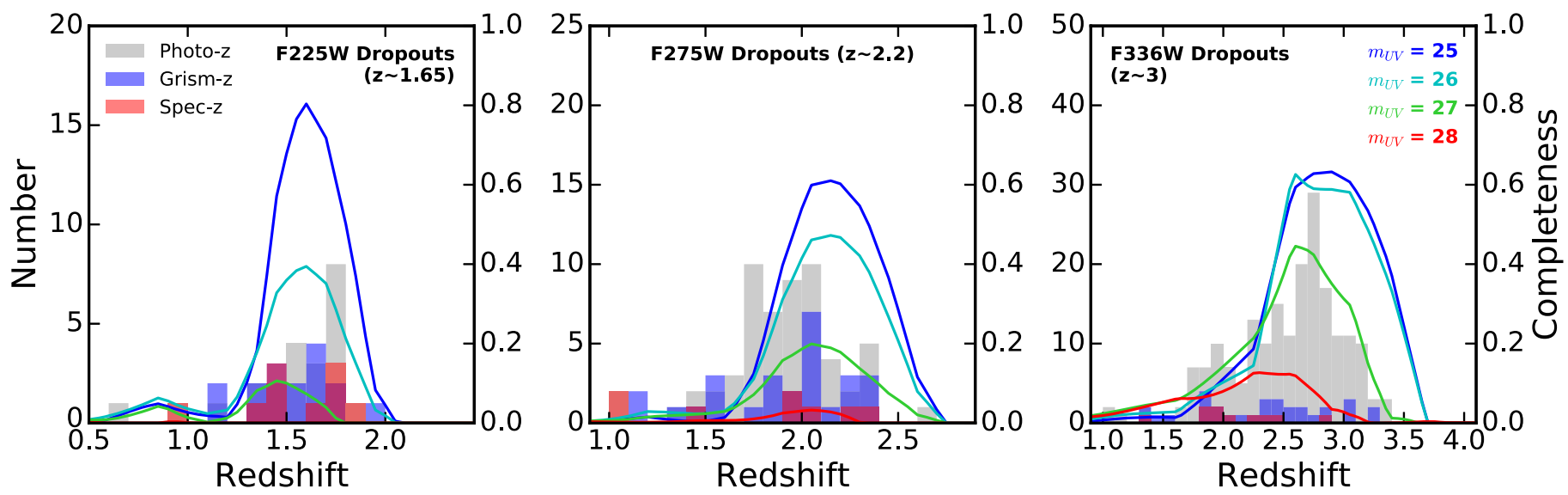

Figure 3. Redshift distribution of the selected-dropout samples. The gray, blue, and red histograms show the distribution of the photometric, grism, and spectroscopic redshifts for the dropout samples, respectively. The curves show the completeness associated with the corresponding dropout criteria for a range of observed UV magnitudes, as computed from our completeness simulations (Section 3). The right-hand axis gives the scale for the completeness values.

defined as

$$
v_{\mathrm{eff}}(M, z)=\frac{d V_{\text {comov }}}{d z d \Omega}(z) \cdot S\left(M, z, r_{1 / 2}\right) \cdot \Omega,
$$

where $S\left(M, z, r_{1 / 2}\right)$ is the selection function, which defines the efficiency of the selection criterion at a given redshift $z$ as a function of the absolute UV magnitude $M$ for a given half-light radius $r_{1 / 2}$ derived from the completeness simulations in Section 3 , and $\Omega$ is the solid angle surveyed. The measurement error associated with the absolute UV magnitude $\left(\sigma_{M, i}\right)$ is modeled as a Gaussian:

$$
N\left(M \mid\left\{M_{i}, \sigma_{i}\right\}\right)=\frac{1}{\sqrt{2 \pi} \sigma_{i}} \exp \left[-\left(\frac{\left(M-M_{i}\right)^{2}}{2 \sigma_{M, i}^{2}}\right)\right] .
$$

In the MLE formalism, the Schechter function normalization $\phi^{\star}$ is calculated after finding the best-fit values for $\alpha$ and $M^{\star}$ :

$$
\phi^{\star}=\frac{N}{\iint_{-\infty}^{M_{\mathrm{lim}}(z)} \phi(M) \cdot v_{\mathrm{eff}}(M, z) \cdot d M d z} .
$$

We construct the $\log$-likelihood function, $\ln \mathcal{L}=\sum_{i=1}^{N}$ $\ln P\left(M_{i}\right)$, where the probability for each source to be detected in the sample, $P\left(M_{i}\right)$, is computed using Equation (5). We maximize the log-likelihood function (alternatively, minimize the negative log-likelihood function). Once the best-fit values for slope $\alpha$ and characteristic magnitude $M^{\star}$ are obtained, the normalization $\phi^{\star}$ is calculated.

Furthermore, in order to properly quantify the uncertainties on our best-fit parameters, we perform a Markov Chain Monte Carlo analysis (MCMC). We probe the full posterior distribution for the free parameters in LF fitting using the Python package emcee (Foreman-Mackey et al. 2013). We implement the Affine-Invariant Ensemble Sampler in emcee, initialized at the best-fit parameters. The uncertainties on our LF parameters are obtained from the distribution of the Markov chain, after discarding the burn-in period.

\section{Results}

\subsection{Rest-frame UV LFs at $z \sim 1.5-3$}

Using the modified MLE-fitting procedure described in Section 4, we fit a rest-frame UV LF for the dropout as well as photometric redshift-selected samples from Section 2. The photometric redshift samples selected over the same redshift ranges as those covered by the dropout samples allows us to verify the robustness of our LF fits.

Ideally, one would use the full sample selected down to the faintest magnitudes possible within the survey capabilities. However, near the survey limit, the incompleteness rises significantly and the correction applied to the effective volumes tends toward considerably large values. In order to avoid using sources with corrections that are too large, we choose to truncate the sample where the effective volume correction rises above $75 \%$ of the correction at the bright end, which further reduces our sample size. Table 1 reports the final sample sizes used to fit the LFs.

\subsection{1. $U V$ LF at $1.4<z<1.9$}

The F225W dropout selection criterion results in a sample of 23 galaxies from the UVUDF catalog. Due to the small sample size, we choose not to fit an LF for the F225W dropouts. The corresponding photometric redshift sample $(1.4<z<1.9)$, however, consists of 202 galaxies-sufficient to properly fit an LF. We use $\mathrm{F} 435 \mathrm{~W}$ as the rest- $1500 \AA$ magnitude and the effective volumes corrected using the selection functions from Section 3. The resulting best-fit parameters are reported in Table 1, and the LF is plotted in the bottom-left panel of Figure 4 along with the results from recent literature.

The UV LFs available from the literature at this redshift already show a considerable spread in their parameters. Our best-fit UV LF expects a higher number density at the bright end compared to other LFs. This can be inferred from the high $M^{\star}$ value we find for our best-fit LF. However, it is important to point out that the area covered by the UVUDF survey is small ( $7.3 \mathrm{arcmin}^{2}$ ), which leads to high cosmic variance. The bright end of the LF is particularly prone to this, given the small number statistics. The faint-end slope of our best-fit LF is also considerably shallow compared to other LFs at similar 
Table 1

Best-fit Parameters for UV LFs

\begin{tabular}{|c|c|c|c|c|c|c|}
\hline Redshift & Sample Selection & $M_{\mathrm{lim}, \mathrm{UV}}$ & $N^{\mathrm{a}}$ & $\alpha$ & $M^{\star}$ & $\overline{\log \phi^{\star}}$ \\
\hline \multicolumn{7}{|c|}{ LBG Dropout Samples } \\
\hline$z \sim 1.65$ & F225W dropouts & -18.46 & 23 & & sample size too small & \\
\hline$z \sim 2.2$ & F275W dropouts & -17.97 & 58 & $-1.31_{-0.75}^{+0.32}$ & $-19.66_{-1.67}^{+0.32}$ & $-2.21_{-1.13}^{+0.18}$ \\
\hline$z \sim 3.0$ & F336W dropouts & -17.37 & 201 & $-1.32_{-0.26}^{+0.07}$ & $-20.61_{-0.92}^{+0.12}$ & $-2.36_{-0.19}^{+0.12}$ \\
\hline \multicolumn{7}{|c|}{ Photometric Redshift Samples } \\
\hline $1.4<z<1.9$ & Photo- $z$ & -15.94 & 202 & $-1.20_{-0.13}^{+0.10}$ & $-19.93_{-0.40}^{+0.25}$ & $-2.12_{-0.19}^{+0.12}$ \\
\hline $1.8<z<2.6$ & Photo-z & -16.30 & 238 & $-1.32_{-0.14}^{+0.10}$ & $-19.92_{-0.44}^{+0.24}$ & $-2.30_{-0.23}^{+0.12}$ \\
\hline $2.4<z<3.6$ & Photo-z & -16.87 & 412 & $-1.39_{-0.12}^{+0.08}$ & $-20.38_{-0.43}^{+0.19}$ & $-2.42_{-0.21}^{+0.11}$ \\
\hline
\end{tabular}

Note.

${ }^{a}$ Sample size after removing any sources with high effective volume correction. See Section 5.1 for details.

redshifts. It is important to note that a higher $M^{\star}$ value also contributes toward flattening the faint-end slope. Our $\alpha$ value still agrees with the F225W dropout LFs from Hathi et al. (2010) and Oesch et al. (2010) within their uncertainties. There is minimal tension between our best-fit and Parsa et al. (2016), as their value is within $<1.5 \sigma$ of ours. However, there is a substantial discrepancy between our result and the Alavi et al. (2016) value of $\alpha=1.56 \pm 0.04$.

\subsection{2. $U V$ LF at $1.8<z<2.6$}

The F275W dropout criterion selects galaxies with $1.8<z<2.6$, where the rest-1500 $\AA$ magnitude is covered by $\mathrm{F} 435 \mathrm{~W}$ for $z<2.2$ and $\mathrm{F} 606 \mathrm{~W}$ for $z>2.2$. For the photometric redshift sample, we use the appropriate rest$1500 \AA$ filter identified using the photometric redshift. However, for the dropout sample, this is not possible due to the lack of individualized redshift information; instead, we use F435W as the rest- $1500 \AA$ filter for the full sample, since it covers rest$1500 \AA$ for the majority of the redshift range (considering the longer tail toward lower redshift). We fit a rest-frame UV LF for the sample of 58 galaxies selected by the $\mathrm{F} 275 \mathrm{~W}$ dropout criterion, with effective volumes corrected according to the corresponding selection function. We also fit an LF using the $1.8<z<2.6$ photometric redshift sample consisting of 238 galaxies. The LF fit using the photometric redshift sample agrees with the dropout sample LF, within the $1 \sigma$ uncertainties. Both $z \sim 2.2$ rest-frame UV LF fits are plotted in the top panel of Figure 4 along with the $68 \%$ confidence regions on the free parameters $\left(\alpha\right.$ and $\left.M^{\star}\right)$ in the inset, and the best-fit parameters are reported in Table 1 .

The nature of the UVUDF observations highlights the ability to detect the faintest galaxies, albeit on a small area. Hence, one of the main goals of this work is to constrain the faint-end slope of the UV LF. At $z \sim 2.2$, we use the UVUDF photometric redshift sample to fit a UV LF faint-end slope of $\alpha=-1.32_{-0.14}^{+0.10}$, which is in good agreement with Parsa et al. (2016) and Sawicki (2012), given their uncertainties. On the other hand, our result is considerably shallower than the estimates from Oesch et al. (2010) and Alavi et al. (2016), who find $\alpha=-1.60 \pm 0.51$ (for their F275W dropout sample) and $\alpha=-1.73 \pm 0.04$, respectively. However, our sample goes $\sim 3$ mag deeper than Oesch et al. (2010), thus providing a tighter constraint on the faint-end slope. Alavi et al. (2016) derive their UV LF using lensed galaxies in the Abell 1689 cluster as well as the Abell 2744 and MACSJ0717 clusters from the Hubble Frontier Fields (HFF). Even though Alavi et al. (2016) go much deeper (down to $M_{\mathrm{UV}}=-13$ ) than the blank-field surveys, there is a possibility of significant systematics affecting their result.

Bouwens et al. (2016) assess the impact of systematic errors on the fits of the LFs derived from lensed galaxy surveys. They find considerable systematic scatter for faint, high magnification sources $(\mu>20)$ dependent on the lens model used, which in turn has a significant impact on the recovered LF. Most dramatically, they find that the faint end of the recovered LF is preferentially steeper than the real value, when the systematic uncertainties in $\mu$ are not accounted for. This systematic could help resolve the tension between our result and Alavi et al. (2016).

\subsection{3. $U V$ LF at $2.4<z<3.6$}

The F336W dropout sample has 201 galaxies and the corresponding photometric redshift sample consists of 412 galaxies. F606W covers the rest-1500 $\AA$ filter for the redshift range selected by the $\mathrm{F} 336 \mathrm{~W}$ dropouts $(2.4<z<3.6)$. Our best-fit values for the Schechter parameters along with the uncertainties for all our fits are reported in Table 1. The bottom right panel of Figure 4 shows the UV LFs for this redshift range, for both dropout and photometric redshift samples, in comparison with the results from recent literature.

Our best-fit rest-frame UV LF at $z \sim 3$ is in excellent agreement with that of Parsa et al. (2016). Our faint-end slope value of $\alpha=-1.39_{-0.12}^{+0.08}$ is considerably shallower than the Reddy \& Steidel (2009) and Oesch et al. (2010) value of $\alpha \sim-1.73$. Similar to the F225W and F275W dropouts, there is significant tension when comparing our result to the Alavi et al. (2016) value of $\alpha \sim-1.94 \pm 0.06$ fit at a slightly lower redshift $z \sim 2.7$.

\subsection{Cosmic Variance}

The error bars shown in Figure 4 already account for the Poisson errors on the number counts. However, given the small field of view of the UVUDF, the number counts are also affected by cosmic variance. This would help explain the discrepancy at the bright end of the lowest redshift $(z \sim 1.65)$ LF compared to 

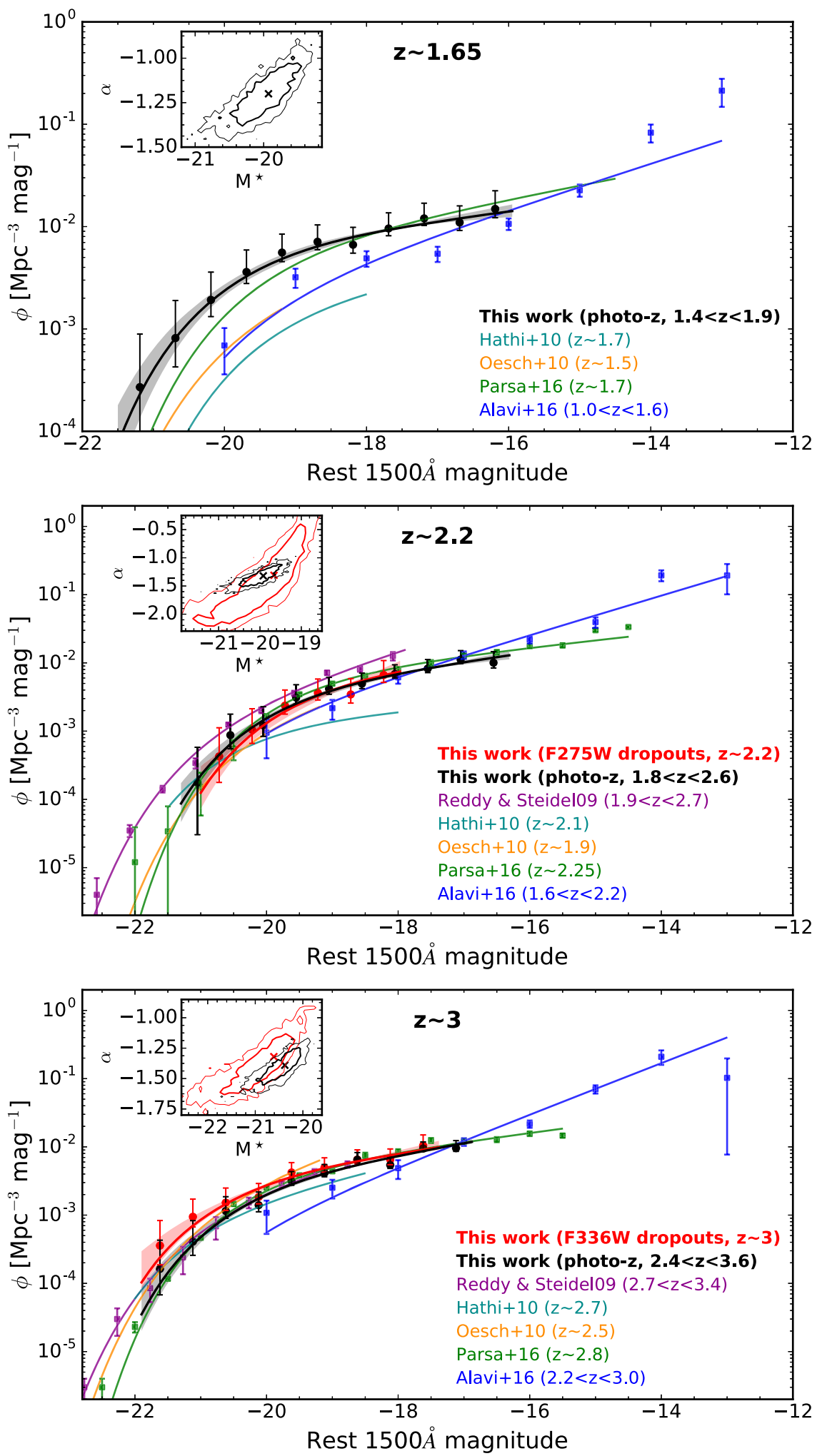

Figure 4. Rest-frame $1500 \AA$ UV luminosity functions at $z \sim 1.7$ (bottom left), $z \sim 2.2$ (top), and $z \sim 3$ (bottom right) measured using UVUDF. The best fits obtained using the dropout samples from UVUDF are shown in red, and the corresponding photometric redshift samples are shown in black. The best-fit parameters for all luminosity function fits are reported in Table 1. The insets show the 68\% (thick) and 95\% (thin) confidence regions for the free luminosity function parameters $\left(\alpha\right.$ and $M^{\star}$ ) obtained from MCMC analysis. The shaded regions denote the $1 \sigma$ confidence regions for the UV luminosity function fits. We also plot the UV luminosity functions at similar redshifts from recent literature for comparison. All LFs have been plotted for the range of magnitudes covered by their samples. 
Table 2

UV Luminosity Density (Not Corrected for Dust)

\begin{tabular}{lrrr}
\hline \hline Redshift & \multicolumn{3}{c}{ UV Luminosity Density $^{\mathrm{a}}$} \\
\cline { 2 - 4 } & $M<-0.03 M_{\mathrm{UV}}^{\star}{ }^{\mathrm{b}}$ & $M<-13$ & $M<-10$ \\
\hline$z \sim 1.65$ & $3.37_{-0.20}^{+0.42}$ & $3.57_{-0.20}^{+0.45}$ & $3.60_{-0.17}^{+0.48}$ \\
$z \sim 2.2$ & $2.42_{-0.18}^{+0.20}$ & $2.65_{-0.15}^{+0.29}$ & $2.69_{-0.15}^{+0.30}$ \\
$z \sim 3.0$ & $2.99_{-0.12}^{+0.33}$ & $3.38_{-0.09}^{+0.35}$ & $3.43_{-0.09}^{+0.40}$ \\
\hline
\end{tabular}

Notes.

${ }^{\mathrm{a}}$ In units of $\times 10^{26} \mathrm{erg} \mathrm{s}^{-1} \mathrm{~Hz}^{-1} \mathrm{Mpc}^{-3}$.

b The $M_{\mathrm{UV}}^{\star}$ value used is from our LF fits using the photometric redshift sample, as reported in Table 1.

other surveys with large coverage. We estimate the cosmic variance for our sample using the Cosmic Variance Calculator v1.02 (Trenti \& Stiavelli 2008.) ${ }^{14}$ For a field of view of $2 ! 7 \times 2 ! 7$, we estimate a fractional error of $0.21,0.21,0.18$ on the number counts of bright $\left(M_{\mathrm{UV}}<-20\right)$ sources in our $1.4<z<1.9,1.8<z<2.6$, and $2.4<z<3.6$ photometric redshift samples, respectively. Similarly, the F275W and F336W LBG dropout samples are affected by a fractional error of $0.22,0.17$ on the number counts of $M_{\mathrm{UV}}<-20$ sources, respectively.

\subsection{UV Luminosity Density}

The faint-end slope of the UV LF determines the relative contribution of faint and bright galaxies to the total cosmic UV luminosity. We use the new estimates derived in Section 5.1 to compute the observed cosmic UV luminosity density (not corrected for dust) as

$$
\rho_{\mathrm{UV}}=\int_{L_{\mathrm{lim}}}^{\infty} L \phi(L) d L=\int_{-\infty}^{M_{\mathrm{lim}}} L(M) \phi(M) d M .
$$

Table 2 reports the UV luminosity density computed integrating down to a variety of luminosity limits. For this calculation, we use the LF fits derived using the photometric redshift samples, due to their smaller statistical uncertainties as well as coverage down to fainter luminosities. The evolution of the UV luminosity density (not corrected for dust) over redshift is shown in Figure 5. All points shown were integrated in a consistent fashion down to $M_{\mathrm{UV}}=-13$ according to Equation (9), using the LF parameters from the cited references along with the reported uncertainties. From $z=0$ to $z=2$, the observed UV luminosity density rises (Arnouts et al. 2005) and peaks around $z \sim 2-3$, after which it slightly declines again (Bouwens et al. 2015; Finkelstein et al. 2015).

Overall, where multiple estimates are available, there is a large scatter in the UV luminosity density. Particularly in the $z \sim 1.5$ to 3.5 range, this scatter is many times larger than the formal errors quoted by some of the surveys, indicating that systematic errors (possibly resulting from the different selection functions and cosmic variance) are not accounted for. Our estimates at $z \sim 2-3$ are within $20 \%$ of the two surveys most similar to ours, Alavi et al. (2016; using lensed galaxies in HFF and Abell 1689) and Parsa et al. (2016; using HUDF without

\footnotetext{
14 http://casa.colorado.edu/ trenti/CosmicVariance.html
}

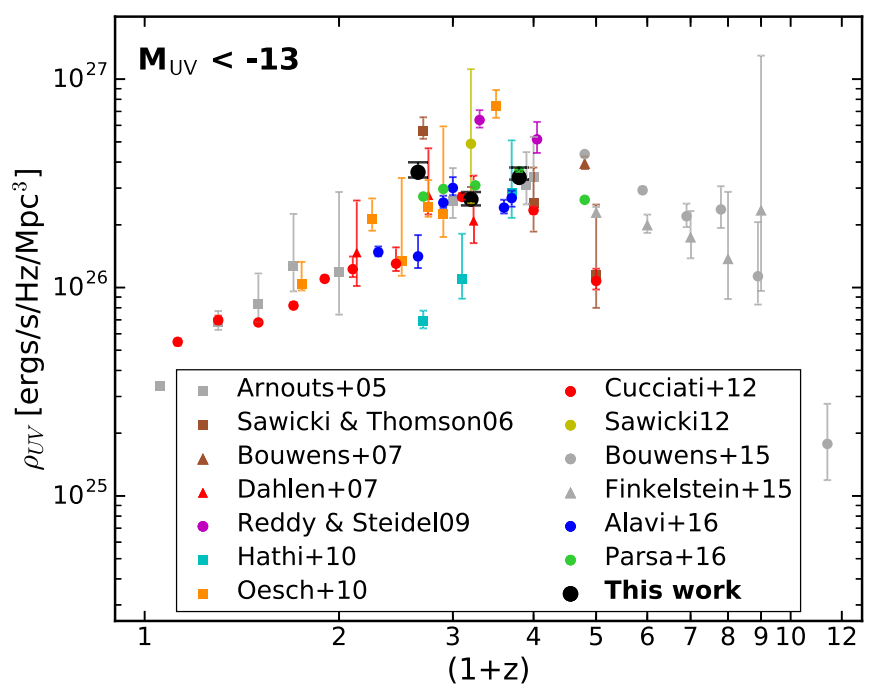

Figure 5. Redshift evolution of the observed UV luminosity density (not corrected for dust). Our points are shown in black and are compared to various rest-frame UV LFs available in the literature at different redshifts. All the points are derived by integrating the rest-frame UV LFs down to $M_{\mathrm{UV}}=-13$, and the errors on the points are estimated using the uncertainties in the LF parameters as reported by the individual references.

the additional NUV coverage). At the lower redshift $z \sim 1.7$, our UV luminosity density estimate is a factor of 2.5 and 1.3 higher than that of Alavi et al. (2016) and Parsa et al. (2016), respectively. This discrepancy is caused by the high number density we find at the bright end compared to the other two LFs. However, we would like to re-emphasize that the coverage area of UVUDF is very small and hence, our result is affected by high cosmic variance.

Using the UV luminosity density, we now compute the cosmic star formation rate density (SFRD) of the universe. Two main assumptions enter this conversion: (i) the correction applied to the UV luminosity to account for interstellar dust attenuation and (ii) the conversion between UV luminosity and SFR (which depends on, e.g., stellar age, SFH, and initial mass function, IMF).

We implement the widely used $I R X-\beta$ relation (Meurer et al. 1999, hereafter M99) to derive the average UV extinction as a function of the observed UV luminosity. The average $\beta$ for our sample is derived as a function of UV luminosity using the $\beta-M_{\mathrm{UV}}$ relation for the appropriate redshift from Kurczynski et al. (2014). The resulting $I R X-\beta-M_{\mathrm{UV}}$ relation quantifies the dust extinction at the observed UV luminosity. For full details on the applied UV dust correction, see Appendix B. The dustcorrected UV luminosity is converted into a star formation rate using the transformations tabulated in Kennicutt \& Evans (2012) (who quote Murphy et al. 2011). The computed SFRDs are reported in Table 3 for the same luminosity ranges used in Table 2.

At $z \sim 2$, we compare our result to the total intrinsic SFRD computed from the UV and IR data in Madau \& Dickinson (2014), $\psi_{\mathrm{UV}+\mathrm{IR}}(z=2.2)=0.127 M_{\odot} \mathrm{yr}^{-1} \mathrm{Mpc}^{-3}$. We measure a dust-corrected UV SFRD of $\psi_{\mathrm{UV}}=0.103 M_{\odot} \mathrm{yr}^{-1} \mathrm{Mpc}^{-3}$ (here, we use the Kennicutt 1998 transformation in order to match the Madau \& Dickinson 2014 analysis) for $M_{\mathrm{UV}}<0.03 M_{\mathrm{UV}}^{\star}$, where $M_{\mathrm{UV}}^{\star}$ is the measurement from our rest-frame UV LF fit using the photometric redshift sample. We find that the derived $\psi_{\mathrm{UV}}$ is approximately a factor of $\sim 1.2$ lower than the total 
Table 3

Star Formation Rate Density (Dust Corrected)

\begin{tabular}{|c|c|c|c|}
\hline \multirow{2}{*}{ Redshift } & \multicolumn{3}{|c|}{ SFR Density ${ }^{\mathrm{a}, \mathrm{b}}$} \\
\hline & $M<0.03 M^{\star c}$ & $M<-13$ & $M<-10$ \\
\hline \multicolumn{4}{|c|}{ Using the Meurer et al. (1999) Relation } \\
\hline$z \sim 1.65$ & $0.094_{-0.008}^{+0.017}$ & $0.097_{-0.008}^{+0.017}$ & $0.098_{-0.008}^{+0.017}$ \\
\hline$z \sim 2.2$ & $0.066_{-0.006}^{+0.008}$ & $0.070_{-0.005}^{+0.010}$ & $0.070_{-0.005}^{+0.010}$ \\
\hline$z \sim 3$ & $0.086_{-0.005}^{+0.013}$ & $0.092_{-0.003}^{+0.013}$ & $0.093_{-0.004}^{+0.012}$ \\
\hline \multicolumn{4}{|c|}{ Using the Castellano et al. (2014) Relation } \\
\hline$z \sim 1.65$ & $0.212_{-0.020}^{+0.036}$ & $0.219_{-0.020}^{+0.038}$ & $0.220_{-0.017}^{+0.036}$ \\
\hline$z \sim 2.2$ & $0.148_{-0.013}^{+0.020}$ & $0.157_{-0.014}^{+0.020}$ & $0.158_{-0.011}^{+0.022}$ \\
\hline$z \sim 3$ & $0.194_{-0.010}^{+0.026}$ & $0.208_{-0.009}^{+0.030}$ & $0.210_{-0.010}^{+0.030}$ \\
\hline \multicolumn{4}{|c|}{ Using the Reddy et al. (2015) Relation } \\
\hline$z \sim 1.65$ & $0.120_{-0.010}^{+0.022}$ & $0.125_{-0.009}^{+0.022}$ & $0.125_{-0.010}^{+0.023}$ \\
\hline$z \sim 2.2$ & $0.084_{-0.007}^{+0.011}$ & $0.089_{-0.007}^{+0.011}$ & $0.090_{-0.007}^{+0.011}$ \\
\hline$z \sim 3$ & $0.110_{-0.004}^{+0.016}$ & $0.118_{-0.005}^{+0.017}$ & $0.119_{-0.005}^{+0.018}$ \\
\hline
\end{tabular}

Notes.

${ }^{\text {a }}$ In units of $M_{\odot} \mathrm{yr}^{-1} \mathrm{Mpc}^{-3}$.

${ }^{\mathrm{b}}$ Using the Kennicutt \& Evans (2012) transformations.

${ }^{c}$ The $M^{\star}$ value used is from our LF fits using the photometric redshift sample, as reported in Table 1.

intrinsic SFRD computed from the UV and IR data in Madau \& Dickinson (2014). At face value, this result suggests that the correction for dust extinction that we apply is underestimated.

We can independently check this result using the $\mathrm{H} \alpha \mathrm{LF}$. For this calculation, we use the Sobral et al. (2013) $z \sim 2.23 \mathrm{H} \alpha$ $\mathrm{LF}$, removing the AGN contribution using the $L_{\mathrm{H} \alpha} / L_{\mathrm{H} \alpha}^{\star}$ versus AGN fraction relation presented in Sobral et al. (2016). To account for dust extinction, we use the luminosity-dependent dust correction from Hopkins et al. (2001), updated for $z \sim 2$ according to the Domínguez et al. (2013) results. For full details on the applied $\mathrm{H} \alpha$ dust correction, see Appendix C.

For a direct comparison, we compute the SFRD by integrating the $\mathrm{H} \alpha$ LF down to an $\mathrm{H} \alpha$ luminosity corresponding to an SFR of $\sim 0.5 M_{\odot} \mathrm{yr}^{-1}$ (i.e., the SFR corresponding to $\left.0.03 M_{\mathrm{UV}, z=2}^{\star}\right)$ and converting the $\mathrm{H} \alpha$ luminosity density into SFRD (using Kennicutt 1998 again, to match the Madau \& Dickinson 2014 analysis). The resulting SFRD is $\psi_{\mathrm{H} \alpha}=0.116$ $M \odot \mathrm{yr}^{-1} \mathrm{Mpc}^{-3}$, more in agreement with the Madau \& Dickinson (2014) UV + IR prediction, and thus, pointing to the dust correction as the main reason for the discrepancy between the SFRD computed from the UV LF alone, and that computed from the UV + IR.

$\mathrm{H} \alpha$ and UV as star formation indicators have been compared using multiwavelength studies, both locally (e.g., Lee et al. 2009, 2011; Domínguez Sánchez et al. 2012; Weisz et al. 2012; Koyama et al. 2015) as well as at high redshifts (e.g., Wuyts et al. 2011; Shivaei et al. 2016). $\mathrm{H} \alpha$ has been shown to be a non-biased SFR indicator that agrees well with the total star formation in normal star-forming galaxies, whereas UV as a star formation indicator by itself is affected by the problem of dust correction. While it is possible that cosmic variance can impact our UV LF, it is important to note that at the redshift we are considering, the bright end of our LF is in very good agreement with other surveys covering large areas. The result, therefore, is not expected to change drastically due to cosmic variance.

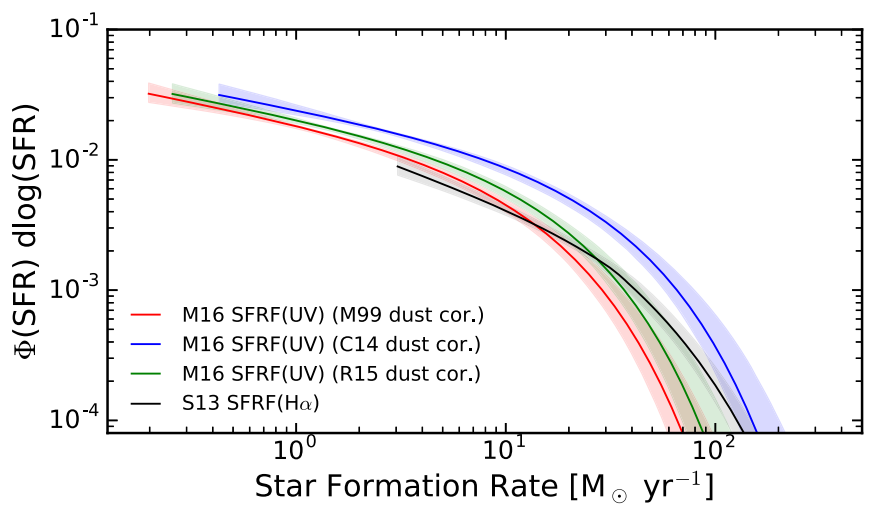

Figure 6. Star formation rate functions derived from the UV luminosity function (this work) with dust corrections applied using the generic Meurer et al. (1999, in red) $I R X-\beta$ relation as well as the Castellano et al. (2014, in blue) and Reddy et al. (2015, in green) relations, which are calibrated at high redshifts $(z \sim 2-3)$. The $\mathrm{H} \alpha$ star formation rate function derived using the Sobral et al. (2013) H $\alpha$ LF and corrected using Hopkins et al. (2001; updated for high redshift using Domínguez et al. 2013 observations) is plotted in black. The UV SFRF corrected using the M99 dust law shows a large discrepancy with the $\mathrm{H} \alpha$ SFRF, particularly at the bright end. Note that the LFs have only been plotted down to the survey limits. See the text for full details on the applied dust corrections.

\section{Discussion}

We further investigate the discrepancies in the volumeaveraged SFR at $z \sim 2$ using the rest-frame UV LF derived in this work and comparing it to the $\mathrm{H} \alpha \mathrm{LFs}$ available in the literature. We start by computing the star formation rate functions (SFRFs) from both the UV and $\mathrm{H} \alpha$ LFs. Similar to the LFs, an SFRF measures the number density of galaxies, but as a function of the star formation rate, instead of luminosity. Converting the LFs to SFRFs requires transforming the luminosities into a star formation rate. As before, we correct the UV LF according to typical dust prescription, the M99 IRX $-\beta$ relation (expanded to an $I R X-\beta-M_{\mathrm{UV}}$ relation), and the $\mathrm{H} \alpha \mathrm{LF}$ according the Hopkins et al. (2001) relation adjusted using Domínguez et al. (2013) results. Both UV and $\mathrm{H} \alpha$ dust-corrected LFs are then converted into SFRFs, using the transformation tabulated in Kennicutt \& Evans (2012; who quote Murphy et al. 2011). ${ }^{15}$

Figure 6 shows the resulting $z \sim 2$ SFRFs, with the SFRF calculated using M99 shown in red and the SFRF from $\mathrm{H} \alpha$ shown in black. The comparison between these two estimates shows that most of the discrepancy originates at the bright end. For SFR $>30 M_{\odot} \mathrm{yr}^{-1}$, the $\mathrm{H} \alpha$ SFRF estimates, for a factor of $\sim 2.5$, more sources compared to the UV SFRF. There is clear tension between the rest-frame $\mathrm{UV}$ and $\mathrm{H} \alpha \mathrm{LFs}$ at $z \sim 2$, under typical assumptions. Hence, one or more of the assumptions made require additional scrutiny. Recalling the main assumptions that enter this analysis:

1. Dust: The observed light (both UV and $\mathrm{H} \alpha$ ) needs to be corrected for interstellar dust absorption before the light can be converted to a star formation rate.

\footnotetext{
15 Specifically, we use:

$$
\begin{gathered}
\mathrm{SFR}_{\mathrm{UV}}\left[M_{\odot} \mathrm{yr}^{-1}\right]=10^{-43.35} \cdot \nu L_{\nu, \mathrm{UV}, \mathrm{corr}}\left[\mathrm{erg} \mathrm{s}^{-1}\right] \\
\quad=0.893 \times 10^{-28} \cdot L_{\nu, 1500, \text { corr }}\left[\mathrm{erg} \mathrm{s}^{-1} \mathrm{~Hz}^{-1}\right] \\
\mathrm{SFR}_{\mathrm{Ha}}\left[M_{\odot} \mathrm{yr}^{-1}\right]=10^{-41.27} \cdot L_{\mathrm{H} \alpha, \operatorname{corr}}\left[\mathrm{erg} \mathrm{s}^{-1}\right] \\
\quad=5.37 \times 10^{-42} \cdot L_{\mathrm{H} \alpha, \text { corr }}\left[\mathrm{erg} \mathrm{s}^{-1}\right] .
\end{gathered}
$$
}


2. Stellar Population Properties: The intrinsic amount of light emitted from a galaxy (star forming or not) depends on stellar population, age, metallicity, and IMF. Consequently, when interpreting the galaxy light as a star formation rate, one has to assume a stellar population model. This assumption can be broken down into finer details: SFH, stellar age, metallicity, and IMF.

It is not straightforward to investigate all of these assumptions simultaneously with only one measurement each of two observables, rest-frame UV and $\mathrm{H} \alpha \mathrm{LFs}$, due to the various degeneracies involved. Here, we individually examine the effects of the most important assumptions: dust and SFHs.

\subsection{Dust Correction}

Correcting for dust is a key step in going from the observed luminosity to the star formation rate. The $I R X-\beta$ relation in M99 was calibrated using local star-forming galaxies. However, the stellar population properties of high-redshift galaxies may be different from local objects, e.g., they are expected to have lower metallicities and younger ages than their local counterparts. This may cause high-redshift galaxies to have intrinsically bluer UV slopes, and using the M99 relation could underestimate the dust content (Wilkins et al. 2012). Recently, amendments to the original M99 IRX $-\beta$ relation have been suggested for high redshift galaxies (e.g., Heinis et al. 2013; Castellano et al. 2014; Reddy et al. 2015). Here, we test two of the recently suggested prescriptions for $z \sim 2$ galaxies: Castellano et al. (2014) and Reddy et al. (2015).

Using a sample of well-studied $z \sim 3$ LBGs, Castellano et al. (2014, hereafter, C14) recently pointed out a systematic offset between the SFR(UV) computed using M99 and those computed from SED fitting. They provide a modification to the $I R X-\beta$ relation by only correcting for the systematic offset: $A_{\mathrm{UV}}=5.32_{-0.37}^{+0.41}+1.99 \beta$, implying a larger correction for dust than when using M99. Reddy et al. (2015, hereafter R15) study a sample of $z \sim 2$ star-forming galaxies with deep optical spectroscopy and multiwavelength photometry. They fit an $I R X-\beta$ slope of $A_{\mathrm{UV}}=4.48+1.84 \beta$ for their $z \sim 2$ sample, which also implies a slightly higher correction for dust than M99. The difference between these two and the M99 relations is highlighted in Appendix B.

In Figure 6, we show the SFRFs computed from the UV LF, but assuming the $\mathrm{C} 14$ (blue curve) and the R15 (green curve) $I R X-\beta$ relation. Both of these dust prescriptions reduce the tension at the highest SFR. The C14 relation, in fact, overcorrects the UV SFRF and only agrees with the H $\alpha$ SFRF at the high-SFR end (SFR $\gtrsim 80 M_{\odot} \mathrm{yr}^{-1}$ ). This is not entirely surprising when considering that $\mathrm{C} 14$ only applied an overall offset to the $I R X-\beta$ relation, which they compute using a sample of high-SFR galaxies (SFR $\sim 100 M_{\odot} \mathrm{yr}^{-1}$ ). Ideally, the $\mathrm{C} 14$ calibration is only valid for the bright end. The UV SFRF corrected using the R15 relation also results in more high-SFR galaxies compared to M99; however, it is still unable to reproduce all of the high-SFR galaxies that are recovered in the $\mathrm{H} \alpha \mathrm{SFRF}$. Thus, a simple tweaking of the dust prescription is not sufficient to solve the discrepancy, as this prescription only measures the average behavior of a galaxy population.

The high-SFR end of the SFRF can be also altered artificially because of the intrinsic scatter in the $I R X-\beta-M_{\mathrm{UV}}$ relation. This effect is partially accounted for in the dust extinction as calculated in Appendix B, where the scatter between $\beta$ and
$M_{\mathrm{UV}}$ is considered (by the $0.2 \ln 10 b^{2} \sigma_{\beta}^{2}$ term in the $A_{\mathrm{UV}}-\beta$ conversion). This correction assumes that the scatter is constant with luminosity and symmetric with respect to the best-fit relation. However, studies have shown that in fact this is not the case, and the scatter increases toward fainter magnitudes, and the distribution around the best-fit becomes skewed, because of the dust-free limit on $\beta$ (e.g., Bouwens et al. 2012; Alavi et al. 2014; Kurczynski et al. 2014). Because of this effect, some faint galaxies may in reality have large dust corrections. A few of these objects would be sufficient to affect the bright end of the SFRF, given its exponential fall off. A proper treatment of the scatter could then reduce the tension between the UV and $\mathrm{H} \alpha$ SFRFs.

There is considerable evidence for the existence of a population of the so-called "Dusty SFGs" (DSFGs; dusty star-forming galaxies), both locally (Goldader et al. 2002; Buat et al. 2005; Burgarella et al. 2005; Howell et al. 2010; Takeuchi et al. 2010) and at high redshifts (Reddy et al. 2010; Heinis et al. 2013; Casey et al. 2014; Ivison et al. 2016). The DSFGs are a population of galaxies that have high star formation rates $\left(\mathrm{SFR}>50 M_{\odot} \mathrm{yr}^{-1}\right)$ and high IR luminosities $\left(L_{\mathrm{IR}} \gtrsim\right.$ few $\left.\times 10^{11} L_{\odot}\right)$. However, due to their high dust content, they have a high $\operatorname{IRX}\left(L_{\mathrm{IR}} / L_{\mathrm{UV}}\right)$ that is offset from the nominal IRX- $\beta$ relation. These galaxies are faint in the UV not because they are intrinsically faint and have low dust content, but instead because they are intrinsically bright and are highly obscured by dust. A generic $I R X-\beta$ relation would underestimate the dust content for these galaxies and hence would result in a deficit of high-SFR sources (SFR $>50 M_{\odot} \mathrm{yr}^{-1}$ ). The shortage of sources in the UV SFRF at the high-SFR end can plausibly be explained by these objects.

\subsection{Star Formation Histories}

The conversion between light (either $\mathrm{H} \alpha$ or UV) and SFR is another key step in the calculation of the SFRFs, which depends critically on the age of the starburst, and therefore on the specific SFH. Until this point, we have assumed the conversion from Kennicutt \& Evans (2012), which implicitly assumes that the SFR has been constant for at least $100 \mathrm{Myr}$. If this is not the case, however, the Kennicutt \& Evans (2012) conversion is not justified, and we have to take into account the fact that the rest-frame UV and the $\mathrm{H} \alpha$ luminosities are sensitive to star formation occurring over different timescales.

In particular, the rest-frame UV is sensitive to star formation occurring over $\sim 100$ s of $\mathrm{Myr}$, whereas the $\mathrm{H} \alpha$ is sensitive to star formation over $\sim 10$ s of Myr. The brightness of a galaxy in the two indicators (i.e., $\mathrm{H} \alpha$ and FUV) depends on the recent SFH. Figure 7 shows the evolution of the UV-to-H $\alpha$ ratio for a variety of SFHs. The $\mathrm{H} \alpha$ output from a galaxy drops on much shorter timescales, after the end of a burst, compared to the non-ionizing UV, which takes longer to react, because of the longer-lived B and A stars that still produce UV photons but have very little ionizing output. For a constant SFH, the rates at which massive stars are formed and die reach an equilibrium after approximately $100 \mathrm{Myr}$, and therefore the UV-to-H $\alpha$ ratio tends to a constant value $\left(\log _{10}\left[\nu L_{\nu}(1500) / L_{\mathrm{H} \alpha}\right] \sim 2\right)$, whereas a burst of star formation would cause the UV-to-H $\alpha$ ratio to scatter toward higher values.

Figure 7 shows how the UV-to-H $\alpha$ ratio evolves for the Bruzual \& Charlot (2003) models with Salpeter (1955), Kroupa (2001), as well as Chabrier (2003) IMFs and metallicities of $Z / Z_{\odot}=0.02,0.2,1$ with three different SFHs: single 


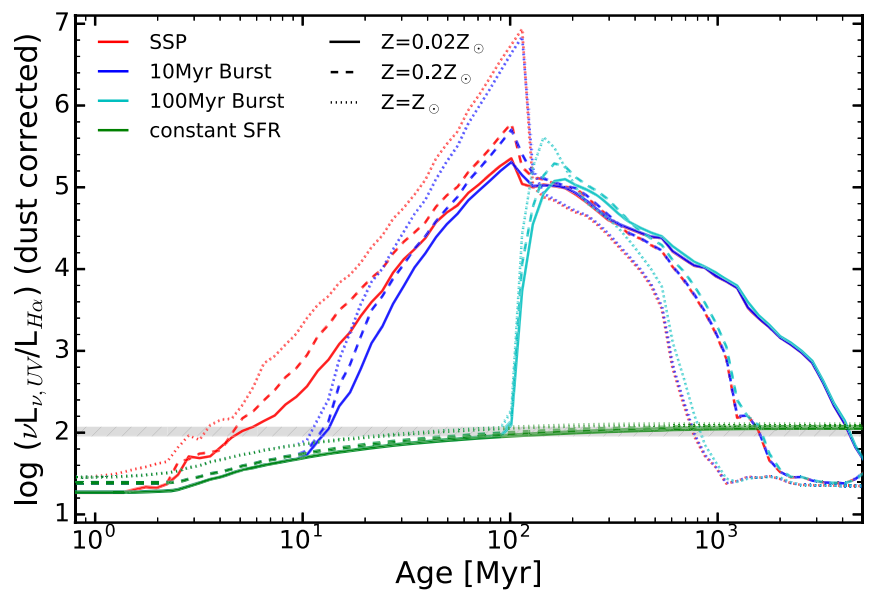

Figure 7. UV-to-H $\alpha$ ratio plotted as a function of age since the onset of star formation for a range of SFHs (single stellar population, single bursts, rising, and constant star formation) computed using the Bruzual \& Charlot (2003) models. The shaded band shows the expected $\log _{10}\left[\nu L_{\nu}(1500) / L_{\mathrm{H} \alpha}\right]$ value for constant star formation, after accounting for a range of metallicities as well as different IMFs. See text for a description of the models.

instantaneous burst (SSP), short bursts of star formation (10 and $100 \mathrm{Myr}$ ), and constant star formation. The main impact of the different IMFs on the UV-to-H $\alpha$ ratio is limited to the values of their slopes at the high-mass end, since both $\mathrm{H} \alpha$ and UV are sensitive to hot massive stars. Moreover, this difference in IMF slopes at the high masses is small enough between the Salpeter (1955, $\alpha=-2.35)$, Kroupa (2001, $\alpha=-2.3$ ), and Chabrier (2003, $\alpha=-2.3$ ) IMFs that the resulting variation in the UV-to-H $\alpha$ ratio is within the linewidths of the curves in Figure 7. For models with constant SFHs, we compute the range of expected UV-to- $\mathrm{H} \alpha$ ratios at an age of $100 \mathrm{Myr}$ (the generic assumption in SFR conversions, e.g., Kennicutt \& Evans 2012). The horizontal band in Figure 7 shows this expectation from the constant SFH case.

The effect of the SFHs on the UV-to-H $\alpha$ ratio has been studied both in simulations (Shen et al. 2013; Hopkins et al. 2014; Domínguez et al. 2015) as well as observations (Boselli et al. 2009; Finkelstein et al. 2011; Lee et al. 2011, 2012; Weisz et al. 2012; Guo et al. 2016). Domínguez et al. (2015) used SFHs derived from hydrodynamical simulations to study the variation of the UV-to- $\mathrm{H} \alpha$ ratio, which they suggest is a useful observable to quantify the "burstiness" of a galaxy's SFH. Using their simulated galaxies, they find that galaxies with low stellar masses $\left(M_{\star} \lesssim 10^{9} M_{\odot}\right)$ are dominated by bursty $\mathrm{SF}$, and have a higher mean value and scatter of the UV-to-H $\alpha$ ratio compared to more massive galaxies. This is a result of energy feedback from star formation being more efficient in low-mass galaxies (Somerville \& Davé 2015).

We can study the trend between the volume-averaged UVto- $\mathrm{H} \alpha$ ratio and the stellar mass by comparing the dustcorrected UV and $\mathrm{H} \alpha$ LFs. To do so, we need to be able to associate a given luminosity (either $\mathrm{H} \alpha$ or UV) with its corresponding halo mass. We use a standard "abundance matching" technique (e.g., Conroy et al. 2006; Guo et al. 2010; Moster et al. 2010; Trenti et al. 2010; Tacchella et al. 2013; Mason et al. 2015) to associate galaxies with given number densities to their corresponding dark-matter halos. The implicit assumption in this step is that there is only one galaxy per darkmatter halo. In practice, we find a relation between the halo mass $\left(M_{h}\right)$ and the observed luminosity (e.g., $\left.L_{\mathrm{UV}}\right)$ by solving

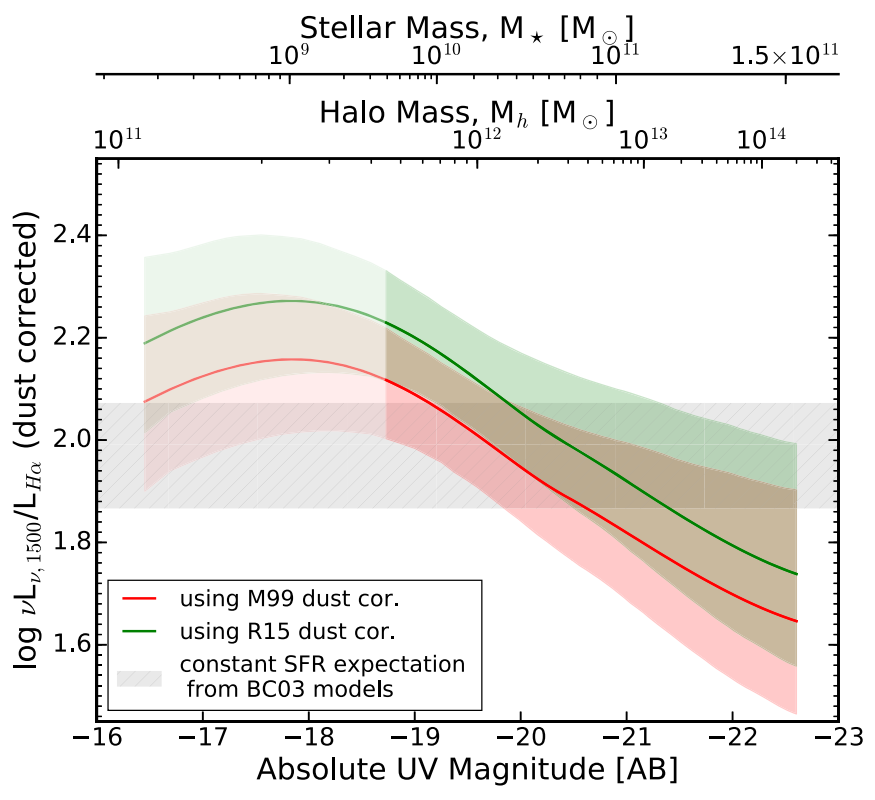

Figure 8. Observed volume-averaged UV-to-H $\alpha$ ratio plotted as a function of the observed UV luminosity using the empirical relations derived from abundance matching the $z \sim 2$ UV LF and Sobral et al. (2013) H $\alpha$ LF, using two UV dust prescriptions: Meurer et al. (1999, red curve) and Reddy et al. (2015, green curve). The horizontal band again shows the expected $\log _{10}\left[\nu L_{\nu}(1500) / L_{\mathrm{H} \alpha}\right]$ value for constant star formation, including a variety of IMFs and metallicities (see text for details). The abundance-matched DM halo mass is plotted on the top axis. Corresponding stellar masses are computed using the $z=2.2$ stellar mass-halo mass relation from Behroozi et al. (2013) and plotted on a parallel axis. The shaded regions show the $1 \sigma$ confidence regions for the measured empirical relation. The darker shaded bands show the range where both UV and $\mathrm{H} \alpha$ LFs have observations, whereas the lighter shaded bands show the range when UV LFs have observations, but the $\mathrm{H} \alpha \mathrm{LF}$ has been extrapolated.

the following equations:

$$
\begin{aligned}
\int_{M_{h}}^{\infty} n\left(M_{h}^{\prime}, z=2.2\right) d M_{h}^{\prime} & =\int_{L_{\mathrm{UV}}}^{\infty} \phi\left(L_{\mathrm{UV}}^{\prime}\right) d L_{\mathrm{UV}}^{\prime} \\
& =\int_{L_{\mathrm{H} \alpha}}^{\infty} \phi\left(L_{\mathrm{H} \alpha}^{\prime}\right) d L_{\mathrm{H} \alpha}^{\prime}
\end{aligned}
$$

where $n\left(M_{h}, z\right)$ is the analytical dark-matter halo mass function from Sheth et al. (2001) computed at $z=2, \phi\left(L_{\mathrm{UV}}\right)$ is the $z \sim 2 \mathrm{UV} \mathrm{LF}$ from this work, and $\phi\left(L_{\mathrm{H} \alpha}\right)$ is the $z=2.23 \mathrm{H} \alpha$ LF from Sobral et al. (2013). By solving Equation (10) we derive the UV and $\mathrm{H} \alpha$ luminosities that correspond to a given dark-matter halo mass, and thus the UV-to-H $\alpha$ ratio that corresponds to that halo mass. We limit this analysis only to luminosities down to which our $z \sim 2$ UV LF sample extends. This does involve extrapolating the $\mathrm{H} \alpha$ LF 1 dex below their observation limit. Also, note that in order to compare to the intrinsic flux ratio, the observed luminosities still need to be corrected for dust. We explore two dust relations (M99 and R15) from the previous section.

Figure 8 shows the main result of this analysis. The horizontal hatched region from Figure 7 is shown again to highlight the expected range of values for $\log _{10}\left[\nu L_{\nu}(1500) / L_{\mathrm{H} \alpha}\right]$ assuming a constant SFH and a range of metallicities $\left(Z / Z_{\odot}=0.02-1\right)$ and different IMFs (Salpeter 1955; Kroupa 2001; Chabrier 2003). The derived volume-averaged UV-to- $\mathrm{H} \alpha$ ratio is not observed to be constant as a function of halo mass, but rather it increases above the value expected for constant $\mathrm{SFH}$, as one moves toward 
Table 4

Polynomial Fits to the Empirical UV-to-H $\alpha$ relation $^{\mathrm{a}}$

\begin{tabular}{|c|c|c|c|c|c|c|c|c|}
\hline UV Dust Correction & $a_{6}$ & $a_{5}$ & $\overline{a_{4}}$ & $a_{3}$ & $\overline{a_{2}}$ & $a_{1}$ & $a_{0}$ & $\sigma^{\mathrm{b}}$ \\
\hline \multicolumn{9}{|c|}{$X=\left(M_{\mathrm{UV}, \mathrm{AB}}-20\right)^{\mathrm{c}}$} \\
\hline Meurer et al. (1999) & $1.46 \mathrm{e}-04$ & $-2.13 \mathrm{e}-05$ & $-2.49 \mathrm{e}-03$ & $-6.37 \mathrm{e}-03$ & $2.21 \mathrm{e}-03$ & $1.41 \mathrm{e}-01$ & 1.95 & 0.154 \\
\hline Reddy et al. (2015) & $1.46 \mathrm{e}-04$ & $-1.19 \mathrm{e}-05$ & $-2.51 \mathrm{e}-03$ & $-6.60 \mathrm{e}-03$ & $1.92 \mathrm{e}-03$ & $1.46 \mathrm{e}-01$ & 2.06 & 0.153 \\
\hline \multicolumn{9}{|c|}{$X=\log \left(M_{h} / 10^{12} M_{\odot}\right)^{\mathrm{d}}$} \\
\hline Meurer et al. (1999) & $-7.61 \mathrm{e}-03$ & $5.58 \mathrm{e}-02$ & $-1.42 \mathrm{e}-01$ & $1.28 \mathrm{e}-01$ & $5.19 \mathrm{e}-02$ & $-2.99 \mathrm{e}-01$ & 2.01 & 0.154 \\
\hline Reddy et al. (2015) & $-7.67 e-03$ & $5.66 \mathrm{e}-02$ & $-1.44 \mathrm{e}-01$ & $1.31 \mathrm{e}-01$ & $5.28 \mathrm{e}-02$ & $-3.10 \mathrm{e}-01$ & 2.12 & 0.153 \\
\hline
\end{tabular}

Notes.

The empirical relation: $\log \left(\nu L_{\nu}(1500) / L_{\mathrm{H} \alpha}\right)=\sum_{i=0}^{N} a_{i} X^{i}$.

b $1 \sigma$ scatter about the mean relation.

c $M_{\mathrm{UV}, \mathrm{AB}}$ range $=(-16.45,-22.60)$.

${ }^{\mathrm{d}} \log \left(M_{h} / M_{\odot}\right)$ range $=(11.05,14.3)$.

the lower halo-mass end $\left(M_{h} \lesssim 10^{12} M_{\odot}\right)$. As seen in Figure 7, the impact of variation in the IMF and/or metallicities on the $\mathrm{UV}$-to-H $\alpha$ ratio is small compared to the variations in the $\mathrm{SFH}$. At face value, the increased ratio at low masses indicates a larger contribution by starbursting objects to the average population of galaxies, as suggested by the larger scatter predicted by Domínguez et al. (2015). The halo mass where this effect seems to be important is below $\sim 5 \times 10^{11} M_{\odot}$, which would correspond to a stellar mass of $\sim 5 \times 10^{9} M_{\odot}$, assuming the stellar mass to halo mass relation from Behroozi et al. (2013). We also provide polynomial fits to the empirical UV-to-H $\alpha$ relation in Table 4 .

It is important to note that the measured empirical UV-to-H $\alpha$ ratio from abundance matching in Figure 8 is very sensitive to the applied dust relation. However, they all exhibit the elevated $\mathrm{UV}$-to-H $\alpha$ ratio. The abundance matching technique matches the cumulative UV and $\mathrm{H} \alpha \mathrm{LFs}$ and hence, it is also sensitive to the systematic differences at the bright end. As noted in the previous section, there is a distinct possibility of the presence of DSFGs in the UVUDF sample that are not being corrected for dust properly by the applied $I R X-\beta$ relation. This impacts the bright end more significantly than the faint end, due to low number statistics. Changing the bright end of the dust-corrected UV LF would offset the UV-to-H $\alpha$ curves in Figure 8 vertically by a significant amount, while changing the overall shape of the curves only minimally. Thus the upturn in the UVto-H $\alpha$ ratio at $M_{h} \lesssim 10^{12} M_{\odot}$ is preserved, although the characteristic stellar mass where this becomes important is somewhat dependent on the specific dust correction used in the analysis.

Figure 8 clearly shows that the observed trend in the UV-to$\mathrm{H} \alpha$ ratio is not constant with the observed UV luminosity and is inconsistent with constant star formation rate at all luminosities, even after accounting for a range of metallicities as well as different canonical IMFs. We also considered the possibility of a non-universal IMF, as introduced by Weidner \& Kroupa (2005), Pflamm-Altenburg et al. (2007, 2009), and Weidner et al. (2011). Based on statistical arguments, Weidner \& Kroupa (2005) produced an integrated galactic initial mass function (IGIMF) that steepens in galaxies with lower SFRs, which could then reproduce a trend similar to that observed in Figure 8. However, the main impact of the SFR-dependent IGIMF occurs at SFRs much lower than those probed by the current analysis $\left(\lesssim 10^{-2} M_{\odot} \mathrm{yr}^{-1}\right.$; Pflamm-Altenburg et al. 2009). In the SFR range $\left(\gtrsim 0.3 M_{\odot} \mathrm{yr}^{-1}\right)$ probed here, the IGIMF is in fact constant with SFR, and thus cannot account by itself for the observed trend in the UV-to-H $\alpha$ empirical relation.

Lastly, the $\mathrm{H} \alpha$ extinction correction we use is rather uncertain and hence, we explore whether the observed trend can be explained uniquely with dust. If dust were the only cause, the required $\mathrm{H} \alpha$ extinction correction would have to be nearly constant with $\mathrm{H} \alpha$ luminosity $(A(\mathrm{H} \alpha) \sim 0.5)$ and thus stellar mass. The latter constraint is, however, inconsistent with observational results. Using a compilation of results from the recent literature, Price et al. (2014) show that the dust extinction increases for brighter, higher-mass galaxies, consistent with earlier results by Garn \& Best (2010).

\section{Conclusions}

NUV coverage of the Hubble UDF provided by the UVUDF enables LBG dropout and photometric redshift selection of galaxies near the peak of cosmic star formation $(z \sim 2-3)$. Additionally, it also enables the study of their rest-frame UV properties and consequently, their star formation properties. Here, we present the rest-frame $1500 \AA$ UV LFs for F225W $(z \sim 1.65)$, F275W $(z \sim 2.2)$, and F336W $(z \sim 3)$ dropout galaxies in the UVUDF selected by the LBG dropout criteria as well as by their photometric redshifts. We develop and execute a suite of completeness simulations to properly correct the effective volumes when fitting the LFs.

Overall, our best-fit rest-frame UV LFs are in good agreement with the recent results from Parsa et al. (2016). We measure faint-end slopes that are within the errors compared to other blank-field surveys (such as Hathi et al. 2010; Oesch et al. 2010). There is a striking discrepancy between our results and those from the Alavi et al. (2016) analysis of lensed galaxies. However, the steep faint end of the LF measured from lensed galaxies could be the result of systematic uncertainties in the lensing modeling. These systematics are particularly important for the most magnified, i.e., intrinsically faintest, sources - those that contribute most to the measurement of $\alpha$ (Bouwens et al. 2016).

At $z \sim 2.2$, using our F275W dropout sample, which covers the absolute UV magnitude range from -22.00 to $-17.97 \mathrm{AB}$ (where the effective volume correction drops to $25 \%$ of that are the bright end), we measure a faint-end slope of $\alpha=-1.31_{-0.75}^{+0.32}$. This is in good agreement with the corresponding photometric redshift sample, which covers a range of -22.00 to $-16.30 \mathrm{AB}$ in absolute UV magnitude, going $\sim 1.5$ mag deeper. When compared 
Table 5

Rest-frame UV LFs Measured via the $V_{\max }$ Estimator $^{\mathrm{a}}$

\begin{tabular}{lccr}
\hline \hline Redshift & $M_{1500}$ & $N^{\mathrm{b}}$ & $\phi\left[\times 10^{-3} \mathrm{Mpc}^{-3} \mathrm{mag}^{-1}\right]^{\mathrm{c}}$ \\
\hline F275W dropouts $z \sim 2.2$ & -18.22 & 14 & \\
& -18.72 & 11 & $6.659_{-1.495}^{+4.158}$ \\
& -19.22 & 15 & $3.447_{-0.873}^{+2.428}$ \\
& -19.72 & 11 & $3.636_{-0.778}^{+2.162}$ \\
& -20.22 & 5 & $2.364_{-0.589}^{+1.639}$ \\
& -20.72 & 2 & $1.049_{-0.388}^{+1.079}$ \\
\hline F336W dropouts $z \sim 3$ & -17.62 & 29 & $0.426_{-0.249}^{+0.693}$ \\
& -18.12 & 28 & $10.461_{-1.618}^{+4.499}$ \\
& -18.62 & 36 & $6.492_{-1.018}^{+2.832}$ \\
& -19.12 & 34 & $6.526_{-0.904}^{+2.514}$ \\
& -19.62 & 34 & $4.953_{-0.703}^{+1.956}$ \\
& -20.12 & 16 & $4.216_{-0.599}^{+1.656}$ \\
& -20.62 & 13 & $1.844_{-0.381}^{+1.060}$ \\
& -21.12 & 8 & $1.522_{-0.349}^{+0.971}$ \\
& -21.62 & 3 & $0.945_{-0.276}^{+0.768}$ \\
& & $0.357_{-0.170}^{+0.474}$ \\
\hline
\end{tabular}

Photometric Redshift Samples

\begin{tabular}{|c|c|c|c|}
\hline \multirow[t]{11}{*}{$1.4<z<1.9$} & -16.19 & 22 & $14.897_{-2.692}^{+7.485}$ \\
\hline & -16.69 & 26 & $10.961_{-1.789}^{+4.975}$ \\
\hline & -17.19 & 32 & $12.028_{-1.764}^{+4.904}$ \\
\hline & -17.69 & 30 & $9.588_{-1.451}^{+4.034}$ \\
\hline & -18.19 & 23 & $6.649_{-1.147}^{+3.189}$ \\
\hline & -18.69 & 25 & $7.112_{-1.177}^{+3.272}$ \\
\hline & -19.19 & 20 & $5.579_{-1.032}^{+2.868}$ \\
\hline & -19.69 & 13 & $3.606_{-0.827}^{+2.299}$ \\
\hline & -20.19 & 7 & $1.921_{-0.600}^{+1.670}$ \\
\hline & -20.69 & 3 & $0.814_{-0.389}^{+1.081}$ \\
\hline & -21.19 & 1 & $0.270_{-0.223}^{+0.621}$ \\
\hline \multirow[t]{10}{*}{$1.8<z<2.6$} & -16.55 & 28 & $10.051_{-1.640}^{+4.560}$ \\
\hline & -17.05 & 44 & $11.191_{-1.419}^{+3.946}$ \\
\hline & -17.55 & 42 & $8.255_{-1.059}^{+2.945}$ \\
\hline & -18.05 & 40 & $6.873_{-0.899}^{+2.500}$ \\
\hline & -18.55 & 29 & $4.958_{-0.761}^{+2.117}$ \\
\hline & -19.05 & 24 & $4.173_{-0.705}^{+1.960}$ \\
\hline & -19.55 & 18 & $3.101_{-0.605}^{+1.682}$ \\
\hline & -20.05 & 7 & $1.217_{-0.380}^{+1.058}$ \\
\hline & -20.55 & 5 & $0.874_{-0.323}^{+0.899}$ \\
\hline & -21.05 & 1 & $0.175_{-0.145}^{+0.403}$ \\
\hline \multirow[t]{10}{*}{$2.4<z<3.6$} & -17.12 & 70 & $9.580_{-0.998}^{+2.776}$ \\
\hline & -17.62 & 87 & $9.451_{-0.851}^{+2.367}$ \\
\hline & -18.12 & 59 & $5.413_{-0.584}^{+1.623}$ \\
\hline & -18.62 & 70 & $6.472_{-0.640}^{+1.780}$ \\
\hline & -19.12 & 48 & $4.142_{-0.495}^{+1.376}$ \\
\hline & -19.62 & 38 & $3.162_{-0.424}^{+1.180}$ \\
\hline & -20.12 & 17 & $1.399_{-0.280}^{+0.780}$ \\
\hline & -20.62 & 14 & $1.152_{-0.254}^{+0.708}$ \\
\hline & -21.12 & 5 & $0.408_{-0.151}^{+0.420}$ \\
\hline & -21.62 & 2 & $0.163_{-0.095}^{+0.265}$ \\
\hline
\end{tabular}

Notes.

${ }^{a}$ The LF fitting is done using the modified MLE technique, which does not require binning and consequently, does not use these numbers explicitly. These numbers are provided for ease of plotting of our data.

${ }^{\mathrm{b}}$ Raw number counts in the luminosity bins.

${ }^{\mathrm{c}}$ Completeness-corrected number densities in the luminosity bins. with results from the literature, we find good agreement with Parsa et al. (2016) as well as Oesch et al. (2010) and Sawicki (2012), given the uncertainties on their result. At $z \sim 1.65$, our best-fit LF estimates a higher number density at the bright end in comparison to other results from the literature. However, due to the small area covered by the UVUDF, this sample is affected by high cosmic variance. For both F275W and F336W dropouts, the LFs measured from the LBG samples agree with those measured from their corresponding photometric redshift samples, within the uncertainties.

We find an observed UV luminosity density (at $M_{\mathrm{UV}}<-13$ ) that is consistent within $20 \%$ of both Alavi et al. (2016) and Parsa et al. (2016) at $z \sim 2-3$. We apply the Meurer et al. (1999) dust relation to correct the UV luminosities and compute the SFRD and find a factor of 2 discrepancy when compared to the total intrinsic star formation rate from UV + IR observations (Madau \& Dickinson 2014). This discrepancy is absent when using a $z \sim 2 \mathrm{H} \alpha \mathrm{LF}$ (Sobral et al. 2013) to compute the SFRD, thus pointing to the dust correction as the main reason for the discrepancy.

We compute the SFRF from the rest-frame UV LF using the generic M99 dust correction. The SFRF corrected according to the M99 relation failed to recover a factor of $\sim 2.5$ higher SFR ( $>30 M_{\odot} \mathrm{yr}^{-1}$ ) sources compared to the $\mathrm{H} \alpha$ SFRF. We find that using the M99 dust correction, which is calibrated using local galaxies, underestimates the dust content in the highredshift $(z \sim 2)$ star-forming galaxies. Using relations calibrated at high redshift, such as those from Castellano et al. (2014) and Reddy et al. (2015), reduces the tension. However, a straightforward tweaking of the $I R X-\beta$ relation is not sufficient to fully resolve the tension. One possibility is the presence of very dusty SFGs, which would not be properly corrected by the IRX $-\beta$ relation because of their offset from the average relation.

Another factor affecting the differences between the UV and $\mathrm{H} \alpha \mathrm{LFs}$ is the burstiness of star formation in galaxies. We use abundance matching of the rest-frame UV and $\mathrm{H} \alpha$ LFs to compute a volume-averaged UV-to-H $\alpha$ ratio-an indicator of "burstiness" in galaxies. We find an increasing UV-to-H $\alpha$ ratio toward low halo masses. We conclude that this trend could be due to a larger contribution from starbursting galaxies at lower masses compared to the high-mass end. This trend is consistent with the expectation from hydrodynamical simulations.

We thank the anonymous referee for providing comments that helped improve the quality of the manuscript. We would like to thank Dan Coe for help with implementing the photometric redshift algorithm (BPZ) into the completeness simulations. Support for HST Program GO-12534 was provided by NASA through grants from the Space Telescope Science Institute, which is operated by the Association of Universities for Research in Astronomy, Inc., under NASA contract NAS526555. M.R. also acknowledges support from an appointment to the NASA Postdoctoral Program at Goddard Space Flight Center. M.B.K. acknowledges support from the National Science Foundation (grant AST-1517226) and from NASA through $H S T$ theory grants (programs AR-12836, AR-13888, AR-13896, and AR-14282) awarded by the Space Telescope Science Institute (STScI), which is operated by the Association of Universities for Research in Astronomy (AURA), Inc., under NASA contract NAS5-26555. 


\section{Appendix A \\ Completeness Functions}

From our simulations, the completeness functions are computed using the fraction of recovered sources as a function of observed magnitude as well as galaxy size. We use the $\mathrm{B}_{435}$ half-light radius as a proxy for the galaxy size. These completeness functions are marginalized over all sizes to obtain completeness just as a function of magnitude, which is used to define the survey magnitude limits. These functions are only used to set the survey magnitude limits when computing the colors of sources not detected in the dropout filter. The effective volumes for computing the LF are fully corrected using the selection functions. Figure 9 shows the completeness functions for the F275W, F336W, and F435W filters. Note that the compact sources have a higher recovery fraction at fainter magnitudes compared to more extended sources.

\section{Appendix B \\ UV Dust Correction}

To correct the observed UV magnitudes, we use the dependence of dust extinction $\left(A_{\mathrm{UV}}\right)$ on the UV slope $\beta$, also known as the $I R X-\beta$ relation. Through the analysis, we implement the $I R X-\beta$ relation $\left(A_{\mathrm{UV}}=a+b \cdot \beta\right)$. We use multiple published fits for this relation: (i) the original Meurer et al. (1999; M99) calibration using local star-forming galaxies: $[a, b]=[4.43,1.99]$, (ii) the Castellano et al. (2014) calibration using high-SFR LBGs at $z \sim 3:[a, b]=\left[5.32_{-0.37}^{+0.41}, 1.99\right]$, and (iii) the Reddy et al. (2015) calibration using $z \sim 2$ starforming galaxies: $[a, b]=[4.48,1.84]$.

Furthermore, the UV slope $\beta$ parameters are estimated as a function of the observed absolute UV magnitudes with a $\beta-M_{\mathrm{UV}}$ relation. The distribution of $\beta$ for the $z \sim 2$ galaxies as a function of the absolute UV magnitudes is assumed using a parametric form, following Trenti et al. (2015) and Mason et al. (2015):

$$
\langle\beta\rangle=\left\{\begin{array}{c}
\left(\beta_{M_{0}}(z)-c\right) \exp \left[-\frac{\frac{d \beta}{d M_{\mathrm{UV}}}(z)\left[M_{\mathrm{UV}}-M_{0}\right]}{\beta_{M_{0}}(z)-c}\right]+c, \\
\quad \text { if } M_{\mathrm{UV}} \geqslant M_{0} \\
\frac{d \beta}{d M_{\mathrm{UV}}}(z)\left[M_{\mathrm{UV}}-M_{0}\right]+\beta_{M_{0}}(z), \\
\quad \text { if } M_{\mathrm{UV}}<M_{0}
\end{array},\right.
$$

where $c$ is the dust-free $\beta$. This relation avoids the unphysical negative values of $A_{\mathrm{UV}}$, while also avoiding unphysical discontinuities near magnitudes where the relation approaches dust-free $\beta$. The parameters $M_{0}, \beta_{M_{0}}, d \beta / d M_{\mathrm{UV}}$, and $\sigma_{\beta}$ define the $M_{\mathrm{UV}}-\beta$ relation and are constrained observationally. We use the results from Kurczynski et al. (2014), who derive this relation for $1<z<8$ galaxies using the UVUDF. For $z \sim 2$, the applied values are $M_{0}=-19.5, \beta_{M_{0}}=-1.71, d \beta / d M_{\mathrm{UV}}=-0.09$, and $\sigma_{\beta}=0.36$.

Moreover, assuming a Gaussian distribution of $\beta$ with a dispersion $\sigma_{\beta}$ gives the average extinction: $\left\langle A_{\mathrm{UV}}\right\rangle=$ $a+0.2 \ln (10) b^{2} \sigma_{\beta}^{2}+b\langle\beta\rangle$, where $b$ is the slope of the IRX $-\beta$ relation (Tacchella et al. 2013; Mason et al. 2015).
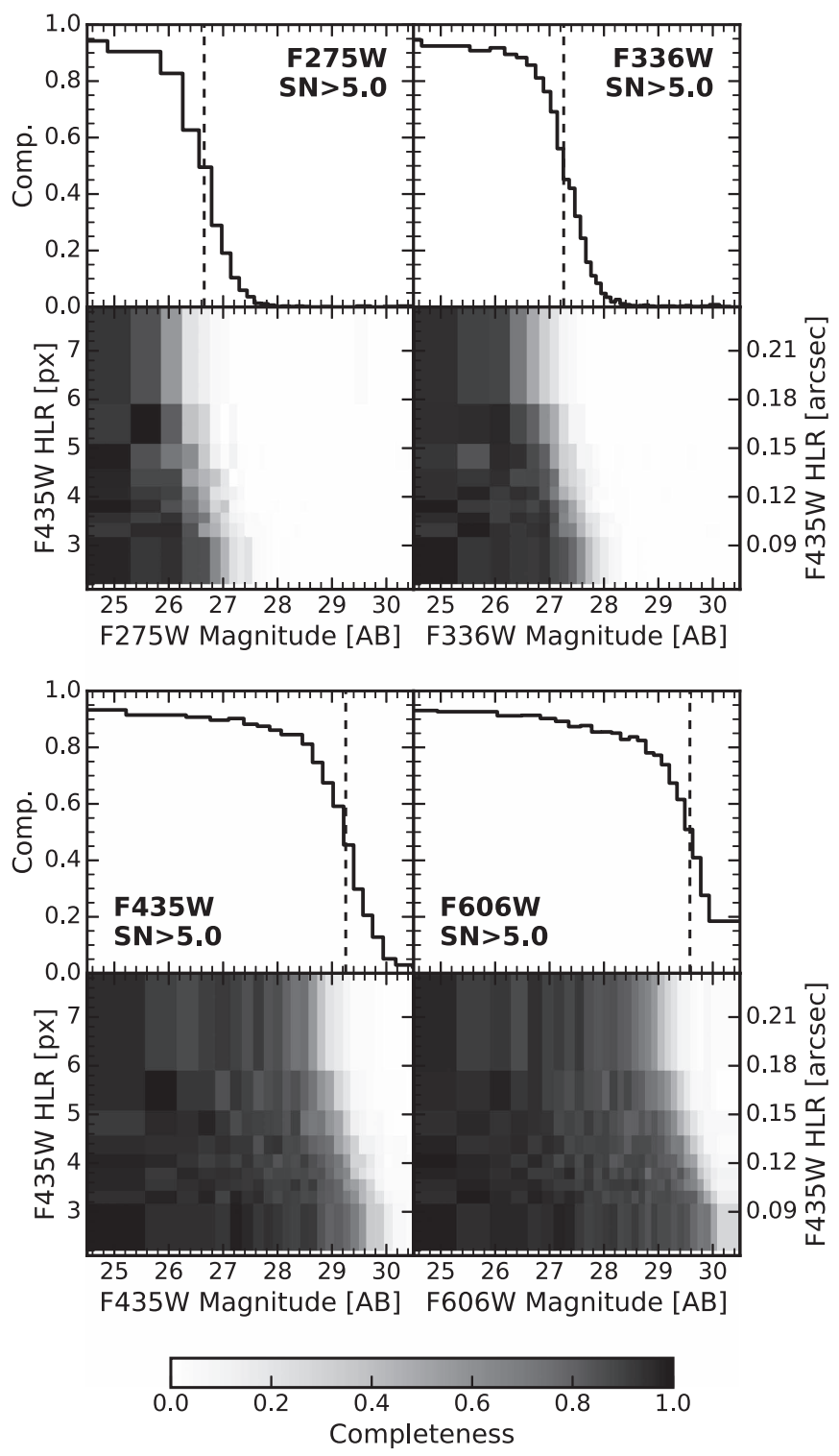

Figure 9. Completeness functions for the F275W, F336W, and F435W filters (from left to right) for sources detected at $>5 \sigma$. The top panel shows the completeness as a function of magnitude only, whereas in the bottom panel, completeness is plotted as a function of magnitude and galaxy size. The dashed lines show where the completeness drops to $50 \%$. The F435W (B-band) halflight radius (HLR) is used as a proxy for galaxy size. From the bottom panels, it is evident that galaxy size has a significant impact on the completenessextended sources are missed more often than the more compact ones, even when they have the same magnitude.

\section{Appendix C \\ H $\alpha$ Dust Correction}

The observed $\mathrm{H} \alpha$ luminosities are corrected for dust extinction by applying the luminosity-dependent dust extinction $\left(A_{\mathrm{H} \alpha}\right)$ reported by Hopkins et al. (2001). They derive an SFR-dependent reddening using a composite of UV, $\mathrm{H} \alpha$ emission line, and FIR data. Most importantly, their dust correction can be applied as a function of attenuated SFR (or light). This is crucial for the analysis here, since the goal is to correct the $\mathrm{H} \alpha \mathrm{LF}$ for dust as a function of the observed $\mathrm{H} \alpha$ luminosity.

However, the Hopkins et al. (2001) relation is derived for local galaxies. In order to scale the relation to match the dust properties of galaxies at $z \sim 2$, we apply a shift such that it 

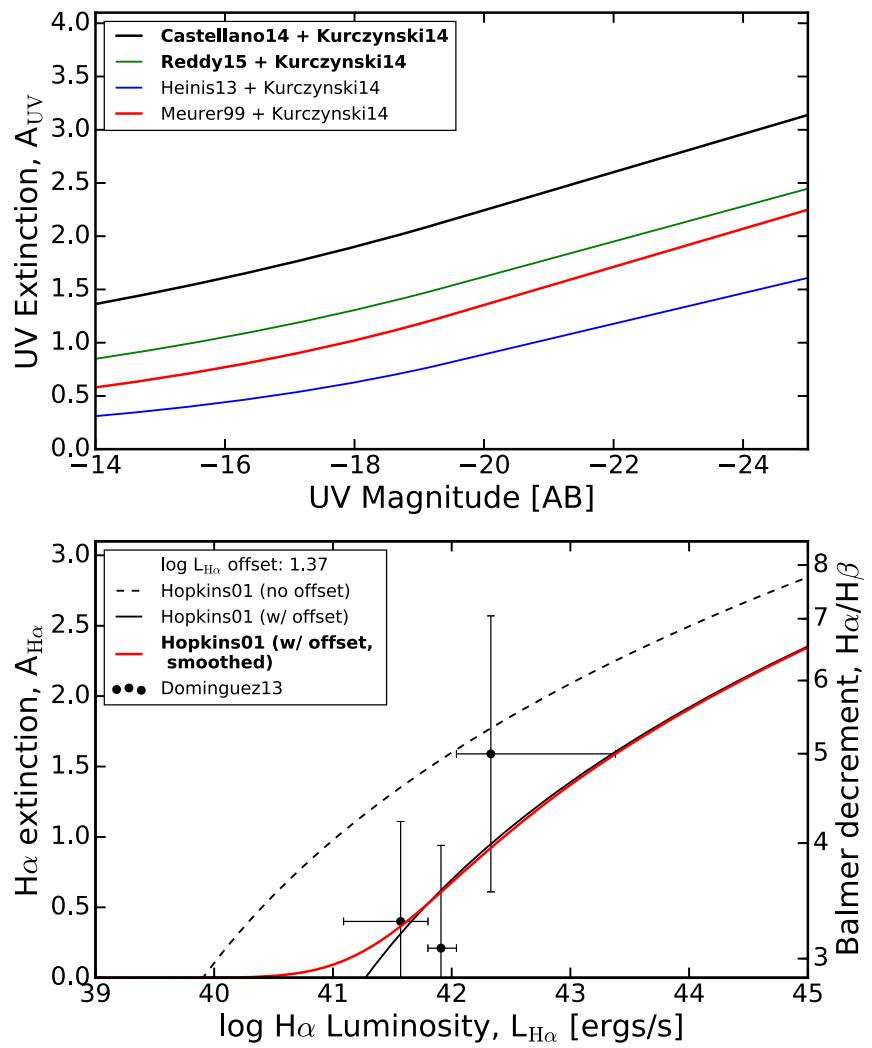

Figure 10. Applied dust extinction correction for our analysis. (Top panel) The dust extinction in rest-frame UV as a function of the observed UV absolute magnitude. The widely used Meurer et al. (1999, M99) relation was calibrated using local star-forming galaxies, whereas the Castellano et al. (2014) relation modifies the M99 relation for higher redshift galaxies. (Bottom panel) The dust extinction in the $\mathrm{H} \alpha$ line as a function of the observed $\mathrm{H} \alpha$ luminosity. The Hopkins et al. (2001) relation for local galaxies is shown with the dashed black curve, whereas the solid curves show the same relation updated for $z \sim 2$ galaxies according to the Domínguez et al. (2013) observations. See text for full details.

matches the Domínguez et al. (2013) observations at $0.7<z<1.5$. This is motivated by the fact that a single relation holds even at higher redshifts, provided that the overall increased star formation (at higher redshift) is accounted for (Sobral et al. 2012). This can be done by applying an offset in the observed luminosity for the $A_{\mathrm{H} \alpha}-L_{\mathrm{H} \alpha}$ relation. This can also be interpreted as the typical dust extinction in $\mathrm{H} \alpha$ not depending on the absolute star formation rate, but rather a relative dependence-how bright (or star-forming) a source is relative to the rest of the galaxy.

Following this justification, we apply an offset of $L_{\mathrm{H} \alpha}=10^{1.37}$ to adjust the Hopkins et al. (2001) local relation to match the Balmer decrement observations of star-forming galaxies at $0.7<z<1.5$ from Domínguez et al. (2013). We also apply a smoothing of 0.5 mag to avoid any discontinuities, as these would, in turn, create unphysical discontinuities in the SFRFs. The bottom panel of Figure 10 shows the dust correction applied to $\mathrm{H} \alpha$ luminosities for our analysis.

\section{References}

Adelberger, K. L., Steidel, C. C., Shapley, A. E., et al. 2004, ApJ, 607, 226 Alavi, A., Siana, B., Richard, J., et al. 2014, ApJ, 780, 143

Alavi, A., Siana, B., Richard, J., et al. 2016, arXiv:1606.00469

Álvarez-Márquez, J., Burgarella, D., Heinis, S., et al. 2016, A\&A, 587, A122

Anders, P., \& Fritze-v. Alvensleben, U. 2003, A\&A, 401, 1063
Arnouts, S., Schiminovich, D., Ilbert, O., et al. 2005, ApJL, 619, L43 Barnes, K. L., van Zee, L., \& Skillman, E. D. 2011, ApJ, 743, 137

Beckwith, S. V. W., Stiavelli, M., Koekemoer, A. M., et al. 2006, AJ, 132, 1729

Behroozi, P. S., Wechsler, R. H., \& Conroy, C. 2013, ApJ, 770, 57

Bell, E. F., \& Kennicutt, R. C., Jr. 2001, ApJ, 548, 681

Benítez, N. 2000, ApJ, 536, 571

Benítez, N., Ford, H., Bouwens, R., et al. 2004, ApJS, 150, 1

Benson, A. J., Frenk, C. S., Baugh, C. M., Cole, S., \& Lacey, C. G. 2003 , MNRAS, 343, 679

Bernard, S. R., Carrasco, D., Trenti, M., et al. 2016, ApJ, 827, 76

Bertin, E., \& Arnouts, S. 1996, A\&AS, 117, 393

Boquien, M., Buat, V., Boselli, A., et al. 2012, A\&A, 539, A145

Boselli, A., Boissier, S., Cortese, L., et al. 2009, ApJ, 706, 1527

Bouwens, R., Broadhurst, T., \& Illingworth, G. 2004, ApJ, 603, 363

Bouwens, R. J., Bradley, L., Zitrin, A., et al. 2014a, ApJ, 795, 126

Bouwens, R. J., Illingworth, G. D., Blakeslee, J. P., \& Franx, M. 2006, ApJ, 653, 53

Bouwens, R. J., Illingworth, G. D., Franx, M., et al. 2009, ApJ, 705, 936

Bouwens, R. J., Illingworth, G. D., Franx, M., \& Ford, H. 2007, ApJ, 670, 928

Bouwens, R. J., Illingworth, G. D., Oesch, P. A., et al. 2010, ApJL, 709, L133 Bouwens, R. J., Illingworth, G. D., Oesch, P. A., et al. 2011, ApJ, 737, 90

Bouwens, R. J., Illingworth, G. D., Oesch, P. A., et al. 2012, ApJL, 752, L5 Bouwens, R. J., Illingworth, G. D., Oesch, P. A., et al. 2014b, ApJ, 793, 115 Bouwens, R. J., Illingworth, G. D., Oesch, P. A., et al. 2015, ApJ, 803, 34

Bouwens, R. J., Oesch, P. A., Illingworth, G. D., Ellis, R. S., \& Stefanon, M. 2016, arXiv:1610.00283

Boylan-Kolchin, M., Weisz, D. R., Johnson, B. D., et al. 2015, MNRAS, 453, 1503

Bruzual, G., \& Charlot, S. 2003, MNRAS, 344, 1000

Buat, V. 1992, A\&A, 264, 444

Buat, V., Donas, J., \& Deharveng, J. M. 1987, A\&A, 185, 33

Buat, V., Iglesias-Páramo, J., Seibert, M., et al. 2005, ApJL, 619, L51

Buat, V., Noll, S., Burgarella, D., et al. 2012, A\&A, 545, A141

Bunker, A. J., Stanway, E. R., Ellis, R. S., \& McMahon, R. G. 2004, MNRAS, 355,374

Bunker, A. J., Wilkins, S., Ellis, R. S., et al. 2010, MNRAS, 409, 855 Burgarella, D., Buat, V., \& Iglesias-Páramo, J. 2005, MNRAS, 360, 1413

Calzetti, D., Armus, L., Bohlin, R. C., et al. 2000, ApJ, 533, 682

Casey, C. M., Scoville, N. Z., Sanders, D. B., et al. 2014, ApJ, 796, 95

Castellano, M., Sommariva, V., Fontana, A., et al. 2014, A\&A, 566, A19

Chabrier, G. 2003, PASP, 115, 763

Coe, D., Benéz, N., Sánchez, S. F., et al. 2006, AJ, 132, 926

Coleman, G. D., Wu, C.-C., \& Weedman, D. W. 1980, ApJS, 43, 393

Conroy, C., Wechsler, R. H., \& Kravtsov, A. V. 2006, ApJ, 647, 201

Cucciati, O., Tresse, L., Ilbert, O., et al. 2012, A\&A, 539, A31

Daddi, E., Dickinson, M., Morrison, G., et al. 2007, ApJ, 670, 156

Dahlen, T., Mobasher, B., Dickinson, M., et al. 2007, ApJ, 654, 172

Dayal, P., \& Ferrara, A. 2012, MNRAS, 421, 2568

de Barros, S., Schaerer, D., \& Stark, D. P. 2014, A\&A, 563, A81

Domínguez, A., Siana, B., Brooks, A. M., et al. 2015, MNRAS, 451, 839

Domínguez, A., Siana, B., Henry, A. L., et al. 2013, ApJ, 763, 145

Domínguez Sánchez, H., Mignoli, M., Pozzi, F., et al. 2012, MNRAS, 426, 330

Ellis, R. S., McLure, R. J., Dunlop, J. S., et al. 2013, ApJL, 763, L7

Finkelstein, K. D., Papovich, C., Finkelstein, S. L., et al. 2011, ApJ, 742, 108

Finkelstein, S. L., Papovich, C., Ryan, R. E., et al. 2012, ApJ, 758, 93

Finkelstein, S. L., Ryan, R. E., Jr., Papovich, C., et al. 2015, ApJ, 810, 71

Fioc, M., \& Rocca-Volmerange, B. 1997, A\&A, 326, 950

Foreman-Mackey, D., Conley, A., Meierjurgen Farr, W., et al. 2013, emcee, Astrophysics Source Code Library, ascl:1303.002

Garn, T., \& Best, P. N. 2010, MNRAS, 409, 421

Goldader, J. D., Meurer, G., Heckman, T. M., et al. 2002, ApJ, 568, 651

Grasha, K., Calzetti, D., Andrews, J. E., Lee, J. C., \& Dale, D. A. 2013, ApJ, 773,174

Grogin, N. A., Kocevski, D. D., Faber, S. M., et al. 2011, ApJS, 197, 35

Guo, Q., White, S., Li, C., \& Boylan-Kolchin, M. 2010, MNRAS, 404, 1111

Guo, Y., Rafelski, M., Faber, S. M., et al. 2016, arXiv:1604.05314

Hathi, N. P., Mobasher, B., Capak, P., Wang, W.-H., \& Ferguson, H. C. 2012, ApJ, 757, 43

Hathi, N. P., Ryan, R. E., Jr., Cohen, S. H., et al. 2010, ApJ, 720, 1708

Hayes, M., Schaerer, D., \& Oumlstlin, G. 2010, A\&A, 509, L5

Heinis, S., Buat, V., Béthermin, M., et al. 2013, MNRAS, 429, 1113

Hermanowicz, M. T., Kennicutt, R. C., \& Eldridge, J. J. 2013, MNRAS, 432, 3097 
Hopkins, A. M., Connolly, A. J., Haarsma, D. B., \& Cram, L. E. 2001, AJ, 122,288

Hopkins, P. F., Kereš, D., Oñorbe, J., et al. 2014, MNRAS, 445, 581

Howell, J. H., Armus, L., Mazzarella, J. M., et al. 2010, ApJ, 715, 572

Iglesias-Páramo, J., Boselli, A., Gavazzi, G., \& Zaccardo, A. 2004, A\&A, 421,887

Inoue, A. K., Shimizu, I., Iwata, I., \& Tanaka, M. 2014, MNRAS, 442, 1805 Ivison, R. J., Lewis, A. J. R., Weiss, A., et al. 2016, arXiv:1611.00762

Jaacks, J., Choi, J.-H., Nagamine, K., Thompson, R., \& Varghese, S. 2012, MNRAS, 420, 1606

Kennedy, R., Frenk, C., Cole, S., \& Benson, A. 2014, MNRAS, 442, 2487

Kennicutt, R. C., \& Evans, N. J. 2012, ARA\&A, 50, 531

Kennicutt, R. C., Jr. 1998, ARA\&A, 36, 189

Koekemoer, A. M., Ellis, R. S., McLure, R. J., et al. 2013, ApJS, 209, 3

Koekemoer, A. M., Faber, S. M., Ferguson, H. C., et al. 2011, ApJS, 197, 36

Koyama, Y., Kodama, T., Hayashi, M., et al. 2015, MNRAS, 453, 879

Kroupa, P. 2001, MNRAS, 322, 231

Kurczynski, P., Gawiser, E., Rafelski, M., et al. 2014, ApJL, 793, L5

Lee, J. C., de Paz, A. G., Tremonti, C., Kennicutt, R. \& Local Volume Legacy Team 2011, in ASP Conf. Ser. 440, UP2010: Have Observations Revealed a Variable Upper End of the Initial Mass Function?, ed. M. Treyer et al. (San Francisco, CA: ASP), 179

Lee, J. C., Gil de Paz, A., Tremonti, C., et al. 2009, ApJ, 706, 599

Lee, K.-S., Ferguson, H. C., Wiklind, T., et al. 2012, ApJ, 752, 66

Lo Faro, B., Monaco, P., Vanzella, E., et al. 2009, MNRAS, 399, 827

Madau, P., \& Dickinson, M. 2014, ARA\&A, 52, 415

Mason, C. A., Trenti, M., \& Treu, T. 2015, ApJ, 813, 21

Mehta, V., Scarlata, C., Colbert, J. W., et al. 2015, ApJ, 811, 141

Menci, N., Fiore, F., \& Lamastra, A. 2012, MNRAS, 421, 2384

Menci, N., Sanchez, N. G., Castellano, M., \& Grazian, A. 2016, ApJ, 818, 90

Meurer, G. R., Heckman, T. M., \& Calzetti, D. 1999, ApJ, 521, 64

Moster, B. P., Somerville, R. S., Maulbetsch, C., et al. 2010, ApJ, 710, 903

Muñoz-Mateos, J. C., Gil de Paz, A., Boissier, S., et al. 2009, ApJ, 701, 1965

Murphy, E. J., Condon, J. J., Schinnerer, E., et al. 2011, ApJ, 737, 67

Nierenberg, A. M., Treu, T., Menci, N., Lu, Y., \& Wang, W. 2013, ApJ, 772,146

Oesch, P. A., Bouwens, R. J., Carollo, C. M., et al. 2010, ApJL, 725, L150

Oke, J. B., \& Gunn, J. E. 1983, ApJ, 266, 713

Parsa, S., Dunlop, J. S., McLure, R. J., \& Mortlock, A. 2016, MNRAS, 456,3194

Pflamm-Altenburg, J., Weidner, C., \& Kroupa, P. 2007, ApJ, 671, 1550

Pflamm-Altenburg, J., Weidner, C., \& Kroupa, P. 2009, MNRAS, 395, 394

Pickles, A. J. 1998, PASP, 110, 863

Planck Collaboration, Ade, P. A. R., Aghanim, N., et al. 2015, arXiv:1502. 01589

Price, S. H., Kriek, M., Brammer, G. B., et al. 2014, ApJ, 788, 86

Rafelski, M., Teplitz, H. I., Gardner, J. P., et al. 2015, AJ, 150, 31

Rafelski, M., Wolfe, A. M., Cooke, J., et al. 2009, ApJ, 703, 2033
Reddy, N. A., Erb, D. K., Pettini, M., Steidel, C. C., \& Shapley, A. E. 2010, ApJ, 712, 1070

Reddy, N. A., Kriek, M., Shapley, A. E., et al. 2015, ApJ, 806, 259

Reddy, N. A., Pettini, M., Steidel, C. C., et al. 2012, ApJ, 754, 25

Reddy, N. A., \& Steidel, C. C. 2009, ApJ, 692, 778

Robertson, B. E., Ellis, R. S., Furlanetto, S. R., \& Dunlop, J. S. 2015, ApJL, 802, L19

Robertson, B. E., Furlanetto, S. R., Schneider, E., et al. 2013, ApJ, 768, 71

Salim, S., Rich, R. M., Charlot, S., et al. 2007, ApJS, 173, 267

Salpeter, E. E. 1955, ApJ, 121, 161

Sawicki, M. 2012, MNRAS, 421, 2187

Sawicki, M., \& Thompson, D. 2006, ApJ, 642, 653

Schechter, P. 1976, ApJ, 203, 297

Shen, S., Madau, P., Guedes, J., et al. 2013, ApJ, 765, 89

Sheth, R. K., Mo, H. J., \& Tormen, G. 2001, MNRAS, 323, 1

Shivaei, I., Kriek, M., Reddy, N. A., et al. 2016, ApJL, 820, L23

Skelton, R. E., Whitaker, K. E., Momcheva, I. G., et al. 2014, ApJS, 214 24

Smit, R., Bouwens, R. J., Labbé, I., et al. 2015, arXiv:1511.08808

Sobral, D., Best, P. N., Matsuda, Y., et al. 2012, MNRAS, 420, 1926

Sobral, D., Smail, I., Best, P. N., et al. 2013, MNRAS, 428, 1128

Sobral, D., Stroe, A., Koyama, Y., et al. 2016, MNRAS, 458, 3443

Somerville, R. S., \& Davé, R. 2015, ARA\&A, 53, 51

Steidel, C. C., Adelberger, K. L., Giavalisco, M., Dickinson, M., \& Pettini, M. 1999, ApJ, 519, 1

Steidel, C. C., Adelberger, K. L., Shapley, A. E., et al. 2003, ApJ, 592, 728

Steidel, C. C., Giavalisco, M., Dickinson, M., \& Adelberger, K. L. 1996, AJ, 112,352

Tacchella, S., Trenti, M., \& Carollo, C. M. 2013, ApJL, 768, L37

Takeuchi, T. T., Buat, V., Heinis, S., et al. 2010, A\&A, 514, A4

Talia, M., Cimatti, A., Pozzetti, L., et al. 2015, A\&A, 582, A80

Teplitz, H. I., Rafelski, M., Kurczy1nski, P., et al. 2013, AJ, 146, 159

Trenti, M., Perna, R., \& Jimenez, R. 2015, ApJ, 802, 103

Trenti, M., \& Stiavelli, M. 2008, ApJ, 676, 767

Trenti, M., Stiavelli, M., Bouwens, R. J., et al. 2010, ApJL, 714, L202

van der Burg, R. F. J., Hildebrandt, H., \& Erben, T. 2010, A\&A, 523, A74

Weidner, C., \& Kroupa, P. 2005, ApJ, 625, 754

Weidner, C., Kroupa, P., \& Pflamm-Altenburg, J. 2011, MNRAS, 412, 979

Weinmann, S. M., Pasquali, A., Oppenheimer, B. D., et al. 2012, MNRAS, 426, 2797

Weisz, D. R., Johnson, B. D., Johnson, L. C., et al. 2012, ApJ, 744, 44

Wilkins, S. M., Gonzalez-Perez, V., Lacey, C. G., \& Baugh, C. M. 2012, MNRAS, 424, 1522

Wuyts, S., Förster Schreiber, N. M., Lutz, D., et al. 2011, ApJ, 738, 106

Wuyts, S., Labbé, I., Förster Schreiber, N. M., et al. 2008, ApJ, 689, 653

Ye, C., Zou, H., Lin, L., et al. 2016, ApJ, 826, 209

Yoshida, M., Shimasaku, K., Kashikawa, N., et al. 2006, ApJ, 653, 988 